\title{
Optimal Segmentation of Three-Dimensional Permanent-Magnet Assemblies
}

\author{
Insinga, Andrea Roberto; Smith, Anders; Bahl, Christian; Nielsen, Kaspar Kirstein; Bjørk, Rasmus
}

Published in:

Physical Review Applied

Link to article, DOI:

10.1103/physrevapplied.12.064034

Publication date:

2019

Document Version

Publisher's PDF, also known as Version of record

Link back to DTU Orbit

Citation (APA):

Insinga, A. R., Smith, A., Bahl, C., Nielsen, K. K., \& Bjørk, R. (2019). Optimal Segmentation of ThreeDimensional Permanent-Magnet Assemblies. Physical Review Applied, 12(6), [064034].

https://doi.org/10.1103/physrevapplied.12.064034

\section{General rights}

Copyright and moral rights for the publications made accessible in the public portal are retained by the authors and/or other copyright owners and it is a condition of accessing publications that users recognise and abide by the legal requirements associated with these rights.

- Users may download and print one copy of any publication from the public portal for the purpose of private study or research.

- You may not further distribute the material or use it for any profit-making activity or commercial gain

- You may freely distribute the URL identifying the publication in the public portal 


\title{
Optimal Segmentation of Three-Dimensional Permanent-Magnet Assemblies
}

\author{
A.R. Insinga $\odot, *$ A. Smith $\odot,{ }^{\dagger}$ C.R.H. Bahl $\odot$, K.K. Nielsen $\odot$, and R. Bjørk $\odot$ \\ Department of Energy Conversion and Storage, Technical University of Denmark - DTU, \\ Anker Engelunds Vej B301, 2800 Kgs. Lyngby, Denmark
}

(Received 31 May 2019; revised manuscript received 24 October 2019; published 13 December 2019)

\begin{abstract}
The optimal segmentation of three-dimensional permanent-magnet systems into uniformly magnetized blocks to generate a desired field is a question that has been studied extensively in recent years. We present a procedure that generates the theoretically optimal shape and magnetization direction of all the magnet segments in the system, given only the total number of blocks as a constraint. Materials of arbitrary magnetic permeability can be included in this optimization framework. As an optimization objective we consider any functional that is linear with respect to the magnetic field distribution. We furthermore assume that all magnetic materials obey a linear constitutive relation. We show that with these assumptions calculating an optimal three-dimensional segmentation is equivalent to determining a centroidal Voronoi tessellation. We use the most-well-known and most-straightforward algorithm for the generation of centroidal Voronoi tessellations, known as "Lloyd's method," which can also be thought of as a fixed-point iteration. We show that the procedure is guaranteed to lead to a configuration that is at least locally optimal with respect to the assumed linear objective functional. However, we present results providing a strong indication that for most design problems applying our technique a few times starting from different random configurations is very likely to lead to the globally optimal solution.
\end{abstract}

DOI: 10.1103/PhysRevApplied.12.064034

\section{INTRODUCTION}

Permanent magnets are central to several scientific and industrial fields [1]. Some of the most-important applications of permanent materials are electromechanical energy-conversion systems [2] such as motors, generators, and actuators [3], as well as magnetic gears and magnetic levitation systems. Additionally, an increasing amount of specialized equipment relies on a precise field distribution generated by permanent magnets. Examples of such devices include multipole magnets [4], wigglers and undulators [5] found in particle accelerators, and nuclearmagnetic-resonance [6] and magnetic-resonance-imaging devices. It is always crucial to optimize such permanentmagnet systems to increase performance, precision, or reliability of the devices and to reduce material cost, weight, and volume.

For practical manufacture, the individual magnet blocks used in a permanent-magnet system need to be uniformly magnetized. Here we introduce an optimization method that can be applied to calculate the optimal way to subdivide a three-dimensional magnetic system into uniformly magnetized blocks. It has been known for some time $[7,8]$ that the optimization of magnetic systems with respect to

*aroin@dtu.dk

†Present address: Villum Foundation, Tobaksvejen 10, Søborg, Denmark linear objectives can be performed by use of the reciprocity theorem [9]. More recently, a method based on this framework was applied to the calculation of the globally optimal segmentation of two-dimensional magnetostatic systems [10].

All the references mentioned above start from the assumption that the magnetic behavior of all the materials can be modeled by a linear constitutive relation. The linear theory of permanent magnets has been discussed extensively [11]. This approximation has been successfully used to analytically derive different important optimality conditions, such as the maximally-energy-efficient remanence vector field that generates a given magnetic field distribution [12]. Moreover, segmented structures have been shown to produce a perfectly uniform field inside a closed cavity $[13,14]$. Even the well-known Halbachcylinder geometry [15] has been constructed starting from the same assumptions [16]. Similarly, the optimality conditions underlying the optimization approach discussed here have been proved to be true only within the linear framework [10].

Our approach is based on the reciprocity theorem, discussed in Sec. II A, which expresses the equivalence between the mutual energies of two magnetostatic systems. We thus consider a different perspective: the requirement in this formalism of a linear objective means that the generated field distribution does not necessarily conform exactly to the desired field distribution. The reason for this is that 
the objective is equivalent to maximizing the intensity of the field along the desired local direction, while not explicitly penalizing the field distortion. Optimization problems aimed at reducing the field distortion would necessarily have to be formulated in terms of a nonlinear objective functional. Linear objectives are, for example, suitable for the typical optimization problems where the intensity of the field must be maximized with a given constraint on the total volume of permanent-magnet material.

While our optimization approach is based on analytically proved optimality conditions, the implementation discussed here relies on numerical methods. For this reason, this technique has some similarities with topologyoptimization algorithms [17-23]. There are no intrinsic limitations to the variety of geometries that can be optimized with the method presented here. The magnetic system may include parts with different magnetic permeability, corresponding to different magnetic materials. However, the permeability distribution must be decided in advance, and thus our method cannot be readily used to determine the optimal shape and position of, for example, iron parts. As for topology-optimization techniques, there is no a priori assumption on the topological configuration of the solution. This also means that the resulting configurations are characterized by curved boundaries, which might have to be simplified to reduce manufacturing difficulties.

Because of its computational complexity, the optimization of three-dimensional magnetic systems is generally challenging for topology-optimization approaches. Using the optimality results mentioned above, we reduce the optimization problem to the problem of calculating a centroidal Voronoi tessellation $[24,25]$. As a consequence of this simplification, our method can be applied to quickly optimize the segmentation of three-dimensional systems. It is also worth stressing that conventional topology-optimization techniques do not offer any insight into why the resulting solution is optimal. In contrast, within the linear framework, the optimality of the solution is seen as a direct consequence of two simply formulated optimality properties.

Building on the approach presented in [10], we consider here the generalization to three-dimensional systems. As shown later, our analysis provides a strong indication that in most cases the calculated segmentations are indeed globally optimal with respect to the objective considered. It is also possible to use our framework to calculate the optimal border between each magnet block and the surrounding air, as long as the magnetic susceptibility of the permanent-magnet material is small. The results of the procedure are optimally segmented structures; an example is shown in Fig. 1.

After introducing our method with simple illustrative cases, we discuss different examples of segmented magnetic systems optimized using the technique presented. We

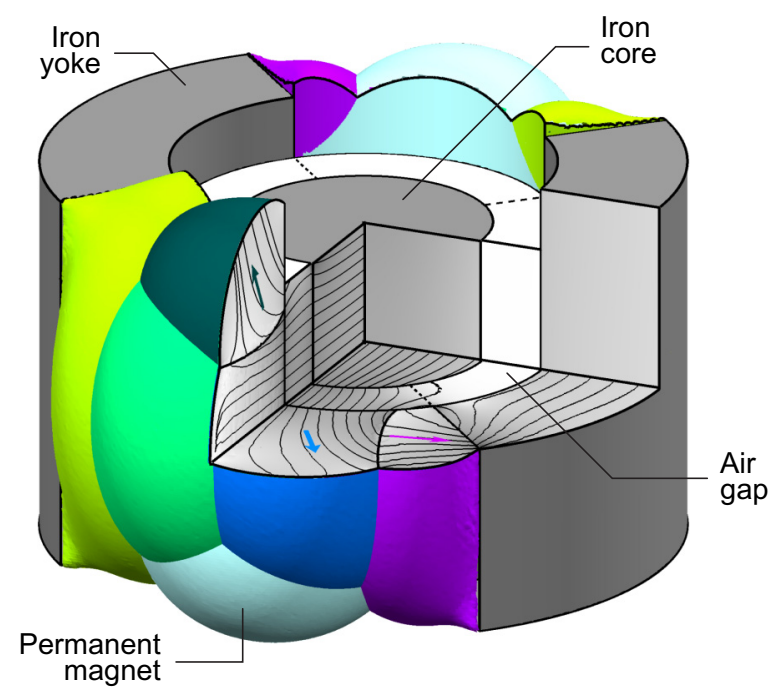

FIG. 1. Example of a magnetic system optimized with the procedure described in the present work. The shape and remanence direction of each permanent-magnet segment are calculated by maximizing the linear objective functional while complying with a constraint on the magnet volume and the number of segments. The directions of the remanence vectors are indicated by the arrows. The flux-density distribution is shown in the cross sections: the field lines indicate the direction, and the darkness of the background indicates higher norm. Further details on this example are given in Sec. III C.

discuss the limitations of the underlying assumptions of our procedure, and finally we consider the structure of the configuration space of possible segmentations and its relation to optimality. The framework introduced here has been implemented into a free software library that is publicly available [26].

\section{METHODS}

\section{A. Framework: the virtual-magnet method}

Our approach is based on the virtual-magnet method [10], an optimization method that relies on the reciprocity theorem [9]. This optimization approach can be applied to optimize the design of a permanent-magnet assembly with respect to any objective functional that is linear with respect to the magnetic field distribution $\mathbf{H}(\mathbf{x})$, where $\mathbf{x}$ represents a given point of space. Any such functional, which we denote by $\mathcal{S}$, can be expressed in integral form:

$$
\mathcal{S}[\mathbf{H}]=\int d V \mathbf{H}(\mathbf{x}) \cdot \mathbf{u}(\mathbf{x}),
$$

where $\mathbf{u}$ denotes the vector field, which can be interpreted as the desired magnetic field distribution. For this reason $\mathbf{u}$ is referred to as the "objective vector field." The integration domain can be reduced to the region where the vector field $\mathbf{u}$ is not zero. As an illustrative example, we consider the two-dimensional optimization problem of maximizing 
(a)

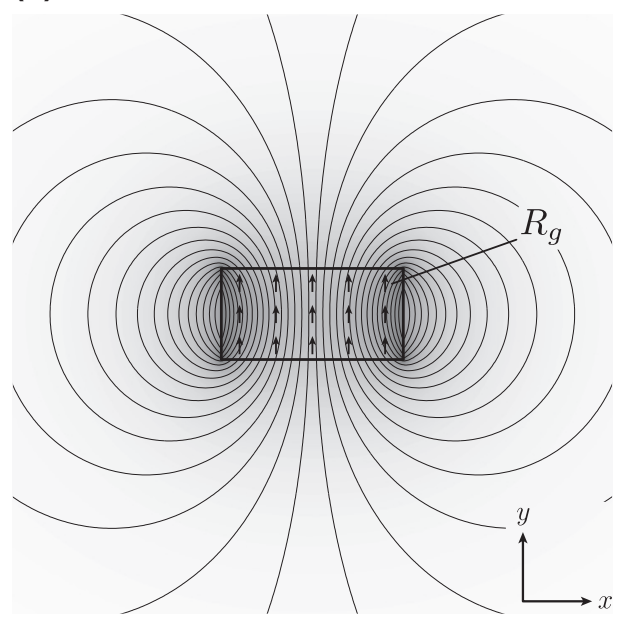

(b)

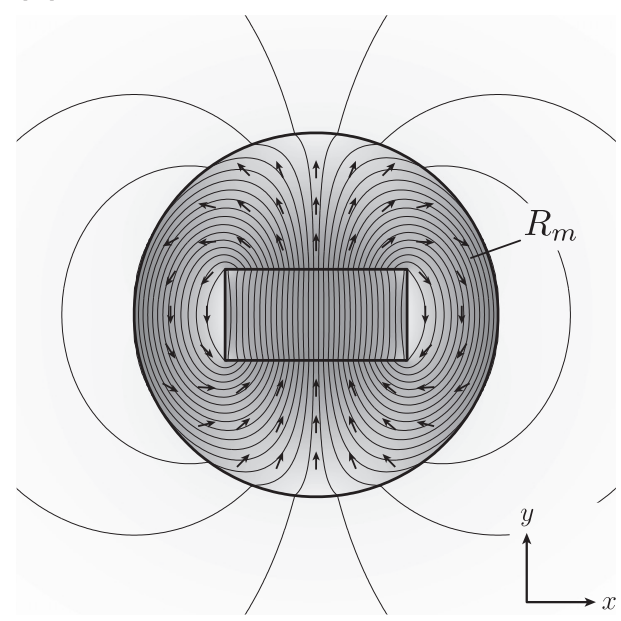

FIG. 2. Optimization of the $y$ component of the field averaged over the central rectangular region $R_{g}$. (a) The virtual system. (b) The real system. In both cases, the remanence is indicated by the black arrows and the flux density is indicated by the field lines. The norm of the flux density is indicated in grayscale, with darker shades indicating higher values. In (b) the magnet region $R_{m}$ is enclosed by the black circle. the $y$ component of the magnetic field averaged over the rectangular region $R_{g}$ indicated in Fig. 2(a). For this purpose the field $\mathbf{u}$, indicated by the black arrows, is a uniform normalized field oriented in the $y$ direction and the norm of $\mathbf{u}$ is zero outside $R_{g}$.

As can be seen, the form expressed by Eq. (1) automatically guarantees linearity of $\mathcal{S}$ with respect to $\mathbf{H}$. This property is expressed by the following equation:

$$
\mathcal{S}\left[\alpha \mathbf{H}_{\alpha}+\beta \mathbf{H}_{\beta}\right]=\alpha \mathcal{S}\left[\mathbf{H}_{\alpha}\right]+\beta \mathcal{S}\left[\mathbf{H}_{\beta}\right],
$$

where $\mathbf{H}_{\alpha}$ and $\mathbf{H}_{\beta}$ are two arbitrary vector fields and $\alpha$ and $\beta$ are two arbitrary real numbers.

It is assumed that for the magnetic behavior of all materials considered in the system there is a linear relation between the flux density $\mathbf{B}$ and the magnetic field $\mathbf{H}$. This relation is expressed in terms of the remanent-flux-density vector $\mathbf{B}_{r}$, and the permeability tensor $\underline{\underline{\mu}}$ :

$$
\mathbf{B}=\underline{\underline{\mu}} \mathbf{H}+\mathbf{B}_{r} .
$$

If a relation of the form given by Eq. (3) holds at every point of the geometry of the system, then the reciprocity theorem [9] can be expressed in terms of $\mathbf{B}_{r}$ :

$$
\int d V \mathbf{H}_{2}(\mathbf{x}) \cdot \mathbf{B}_{r 1}(\mathbf{x})=\int d V \mathbf{H}_{1}(\mathbf{x}) \cdot \mathbf{B}_{r 2}(\mathbf{x}),
$$

where the labels 1 and 2 identify two independent magnetic systems defined over the same region of space, and sharing the same permeability field $\underline{\mu}(\mathbf{x})$, which must also be a symmetric tensor at every point. The system labeled by 1 is the real magnetic system, which we would like to optimize: the magnetic field distribution $\mathbf{H}_{1}(\mathbf{x})$ is the field that should maximize the linear objective functional, and the remanence distribution $\mathbf{B}_{r 1}(\mathbf{x})$ is the remanent flux density that generates $\mathbf{H}_{1}$. On the other hand, the system labeled by 2 is merely an abstraction that provides a powerful way to calculate the optimal remanence distribution $\mathbf{B}_{r 1}$.

If $\mathbf{B}_{r_{2}}$ is replaced by $\mathbf{u}$, the right-hand side of Eq. (4) is identical to the expression for the general linear objective functional $\mathcal{S}$ defined in Eq. (1). Because of the reciprocity theorem (i.e., ultimately because of linearity), the optimal direction of the real remanence $\mathbf{B}_{r 1}$ at any point is aligned with the field $\mathbf{H}_{2}$ generated by a remanence distribution $\mathbf{B}_{r 2}$ that is identical to the desired distribution $\mathbf{u}$. Therefore, it is logical to refer to the system labeled by 2 as the "virtual magnetic system," which is the core of the virtual-magnet method [10].

For the example shown in Fig. 2(a) the direction of the virtual flux density $\mathbf{B}_{2}$ is indicated by field lines, and the norm $B_{2}$ is indicated in grayscale, with darker shades indicating higher values of $B_{2}$. Figure 2(b) shows the corresponding real magnetic system with the same notation. As indicated in Fig. 2(b), the magnet region $R_{m}$ is chosen as a circular region that surrounds $R_{g}$. The remanence distribution $\mathbf{B}_{r 1}$, indicated by the black arrows, is a field of uniform norm aligned at any point of $R_{m}$ with $\mathbf{H}_{2}$.

\section{B. Properties of optimally segmented assemblies}

In practice, magnetic assemblies are realized by a combination of several blocks of permanent-magnet material, each characterized by a remanence distribution that is considered uniform inside its domain. In this section we analyze how to segment a given region $R_{m}$ into blocks with uniform remanence in a way that the linear objective functional is maximized. This procedure consists in determining the shape and remanence direction of each segment, given the number of segments $N$ as a constraint. In this respect the permeability distribution $\underline{\underline{\mu}}(\mathbf{x})$ is assumed to be predetermined. For now we focus on the case where the magnet-design area $R_{m}$, which is also predetermined, is entirely filled by the magnet blocks. 
Let us consider one such block, labeled by $k$. We denote by $R_{m}^{(k)}$ the region of space occupied by this block and by $\mathbf{B}_{r 1}^{(k)}$ the corresponding remanent-flux-density vector. Since we consider magnetic systems where all the blocks are composed of the same permanent-magnet material, the norm of the remanence is assumed to be the same for all blocks, and is denoted by $B_{r}$.

The linearity of the objective functional implies that for several magnet blocks, $\mathcal{S}$ is obtained by adding the individual contributions $\mathcal{S}^{(k)}$ from the different blocks:

$$
\mathcal{S}=\sum_{k=1}^{N} \mathcal{S}^{(k)}=\sum_{k=1}^{N} \int_{R_{m}^{(k)}} d V \mathbf{B}_{r 1}^{(k)} \cdot \mathbf{H}_{2}(\mathbf{x}) .
$$

We introduce the set of $N$ vectors $\mathcal{H}_{2}^{(k)}$ denoting the volume integral of the virtual field over each of the regions $R_{m}^{(k)}$ :

$$
\mathcal{H}_{2}^{(k)}=\int_{R_{m}^{(k)}} d V \mathbf{H}_{2}(\mathbf{x})
$$

As the remanence is uniform over each of the regions $R_{m}^{(k)}$, $\mathcal{S}^{(k)}$ is given by

$$
\mathcal{S}^{(k)}=\mathbf{B}_{r 1}^{(k)} \cdot \mathcal{H}_{2}^{(k)}
$$

We also introduce the $N$ scalar fields $L^{(k)}(\mathbf{x})$, which express how much a point $\mathbf{x}$ contributes to $\mathcal{S}$ if the point is assigned to the $k$ th magnet segment:

$$
L^{(k)}(\mathbf{x})=\mathbf{B}_{r 1}^{(k)} \cdot \mathbf{H}_{2}(\mathbf{x}) .
$$

$\mathcal{S}^{(k)}$ can thus also be expressed as

$$
\mathcal{S}^{(k)}=\int_{R_{m}^{(k)}} d V L^{(k)}(\mathbf{x}) .
$$

Our approach is based on two important implications of the reciprocity theorem, expressed as properties satisfied by an optimal segmentation:

(a) Property 1. Considering the regions $R_{m}^{(k)}$ fixed, the optimal remanence vector $\mathbf{B}_{r 1}^{(k) \text { opt }}$ of a magnet block is aligned with the direction of the virtual field $\mathbf{H}_{2}$ averaged over the region occupied by the block:

$$
\mathbf{B}_{r 1}^{(k) \text { opt }}=B_{r} \hat{\mathcal{H}}_{2}^{(k)},
$$

where $\hat{\mathcal{H}}_{2}^{(k)}$ denotes the unit vector having the same direction as $\mathcal{H}_{2}^{(k)}$. This property follows from Eq. (7).

(b) Property 2. Considering the remanence vectors $\mathbf{B}_{r 1}^{(k)}$ fixed, the optimal subdivision in domains $R_{m}^{(k) \text { opt }}$ is obtained by assigning each point $\mathbf{x} \in R_{m}$ to the block $k$ whose remanence direction is best aligned to the direction $\mathbf{H}_{2}(\mathbf{x})$ of the virtual field at that point:

$$
R_{m}^{(k) \text { opt }}=\left\{\mathbf{x} \in R_{m}: L^{(k)}(\mathbf{x})>L^{\left(k^{\prime}\right)}(\mathbf{x}) \forall k^{\prime} \neq k\right\} .
$$

This property, which follows from Eq. (9), implies that the boundary between two adjacent blocks is composed of points for which the virtual field is equally aligned with the remanence vectors of the two blocks.

It is important to realize that the global optimality of the directions $\mathbf{B}_{r 1}^{(k)}$ with respect to a fixed set of domains $R_{m}^{(k)}$ does not automatically guarantee the optimality of those domains with respect to the set of directions. The converse is also true: global optimality of the domains with respect to a fixed set of directions does not imply the optimality of those directions with respect to the domains. When the two conditions are satisfied simultaneously, the solution is at least locally optimal with respect to the whole configuration space (i.e., directions and domains).

As an illustrative example we again consider the twodimensional system shown in Fig. 2(b). We apply the segmentation procedure with $N=2$ blocks to the portion of $R_{m}$ lying inside the first quadrant of the $x-y$ plane. Because of the second property mentioned above, for a two-dimensional system the optimal border between the two adjacent blocks is given by a set of points where the direction of $\mathbf{H}_{2}$ is constant [10]. We can express the virtual field in terms of its norm, $\mathrm{H}_{2}$, and its azimuthal angle, $\psi$ :

$$
\mathbf{H}_{2}(\mathbf{x})=H_{2}(\mathbf{x})\left[\cos (\psi(\mathbf{x})) \hat{\mathbf{e}}_{x}+\sin (\psi(\mathbf{x})) \hat{\mathbf{e}}_{y}\right],
$$

where $\hat{\mathbf{e}}_{x}$ and $\hat{\mathbf{e}}_{y}$ denote the unit vectors for the $x$ and $y$ coordinates, respectively. The optimal border between the segments is thus given by a level curve of $\psi(\mathbf{x})$. It is assumed that each level curve is a connected set; this assumption is discussed in more detail in Sec. IV A. The level curves are shown as thin black lines in Fig. 3. For Fig. 3(a), the directions of the remanence vectors are chosen arbitrarily: $\mathbf{B}_{r 1}^{(1)}=B_{r} \hat{\mathbf{e}}_{y}$ and $\mathbf{B}_{r 1}^{(2)}=B_{r} \hat{\mathbf{e}}_{x}$, corresponding to $\psi=\pi / 2$ and $\psi=0$, respectively. These vectors are indicated by the blue arrows, and the border between the corresponding optimal magnet domains $R_{m}^{(k) \text { opt }}$ is indicated by the thick blue line. On the optimal border between the two segments, the direction of $\mathbf{H}_{2}$, indicated here by the small black arrows, bisects the angle between $\mathbf{B}_{r 1}^{(1)}$ and $\mathbf{B}_{r 1}^{(2)}$ (i.e., $\psi=\pi / 4)$.

Figure 3(b) shows as red arrows the optimal remanence vectors $\mathbf{B}_{r 1}^{(k)}$ opt for the same regions. These vectors are aligned with the space averages of $\mathbf{H}_{2}$ over the corresponding regions, and are different from the starting vectors shown in Fig. 3(a). The iterative algorithm leading to Fig. 3(c) and ultimately to Fig. 3(d) is the subject of the next section. 
(a)

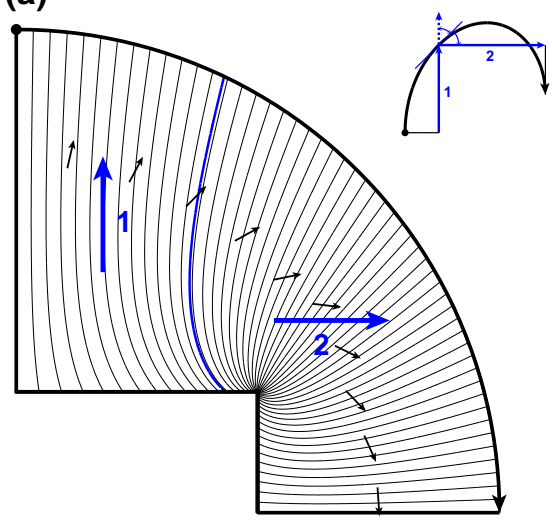

(c)

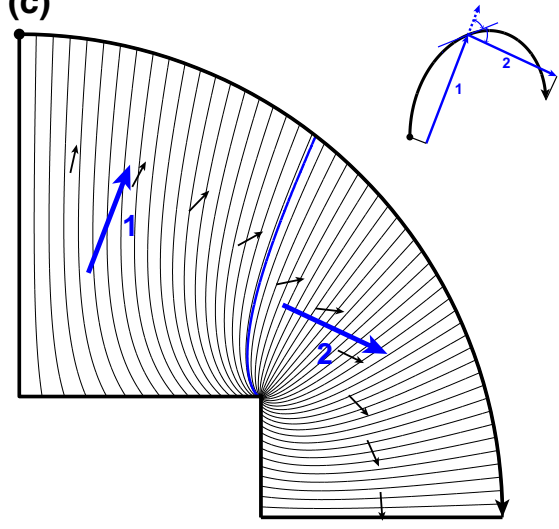

(b)

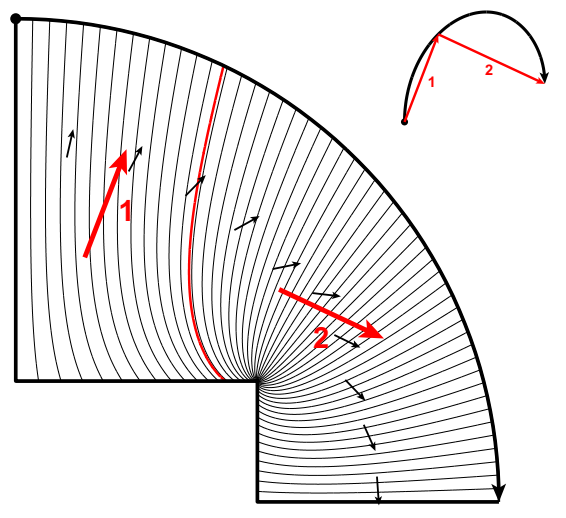

(d)

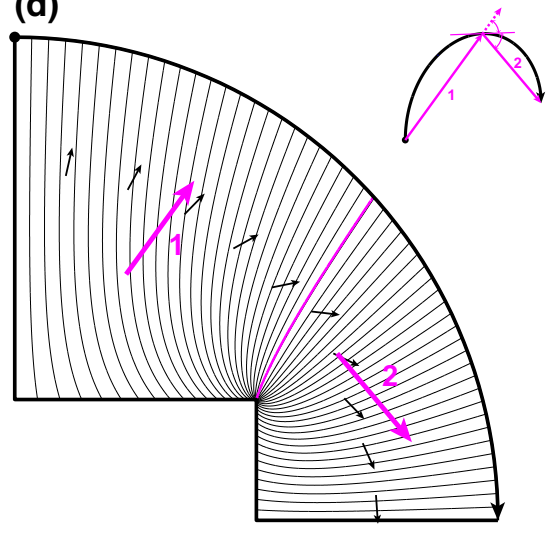

FIG. 3. Optimization procedure with $N=2$ segments applied to the first quadrant of the geometry for the system shown in Fig. 2. The starting directions are arbitrarily set to $\psi=0$ and $\psi=\pi / 2$. The corresponding optimal regions are shown in 3(a). The optimal directions for these regions are shown in 3(b). The two regions are then optimized again from this set of directions, as shown in (c). Eventually, the procedure generates a segmentation satisfying both optimality conditions; that is, the geometry shown in 3(d). The diagrams shown in the upper-right corner of each figure part highlight the analogy with the curve-segmentation problem.

\section{Optimization procedure}

The iterative optimization approach described in this paper is an immediate consequence of the two properties discussed in the previous section. The first property expresses the global optimality of the remanence directions with respect to the reduced configuration space of fixed domains. Therefore, if the directions are updated on the basis of a fixed set of domains, the value of the objective functional $\mathcal{S}$ can only increase. Similarly, if the domains are updated on the basis of a fixed set of directions, the value of $\mathcal{S}$ increases. Starting with an arbitrary set of directions and iteratively updating domains and directions will thus ultimately lead to a locally optimal point where both properties are simultaneously satisfied. As discussed in Sec. II D, this procedure is analogous to Lloyd's algorithm [24,25,27].

The optimization procedure is symbolically illustrated in Fig. 4(a). The horizontal axis represents all the directions of the set of remanence vectors $\mathbf{B}_{r 1}^{(k)}$. The vertical axis corresponds to the set of domains $R_{m}^{(k)}$ of the magnet blocks. The thick red curve indicates configurations for which the remanence directions are optimal with respect to a fixed set of domains. Analogously, the thick blue curve corresponds to configurations for which the domains are optimal for a fixed set of remanence directions. Using a simplified suggestive notation, we express the two properties with the following equations:

$$
\frac{\partial \mathcal{S}}{\partial\left\{\mathbf{B}_{r 1}^{(k)}\right\}}=0 ; \quad \frac{\partial \mathcal{S}}{\partial\left\{R_{m}^{(k)}\right\}}=0 .
$$

The iterative procedures starts with an arbitrary set of directions and the corresponding optimal domains, as indicated by the black dot labeled by the letter $A$ in Fig. 4(a). As mentioned above, this segmentation is globally optimal with respect to the reduced configuration space corresponding to the fixed set of directions. This point corresponds to the configuration shown Fig. 3(a).

The remanence directions are then updated on the basis of the domains, as indicated in Fig. 4(a) by the thin red arrow going from point $A$ to point $B$, which corresponds to Fig. 3(b). The iteration continues by updating the domains on the basis of the new set of directions. This configuration corresponds to point $C$ in Fig. 4(a) and to the geometry shown Fig. 3(c).

The iteration traces a staircase trajectory in the configuration space, terminating at the magenta point labeled by $D$, where both properties are verified at the same time. 
(a)

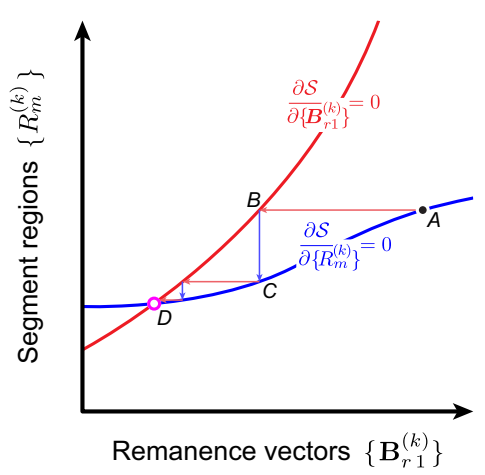

(b)

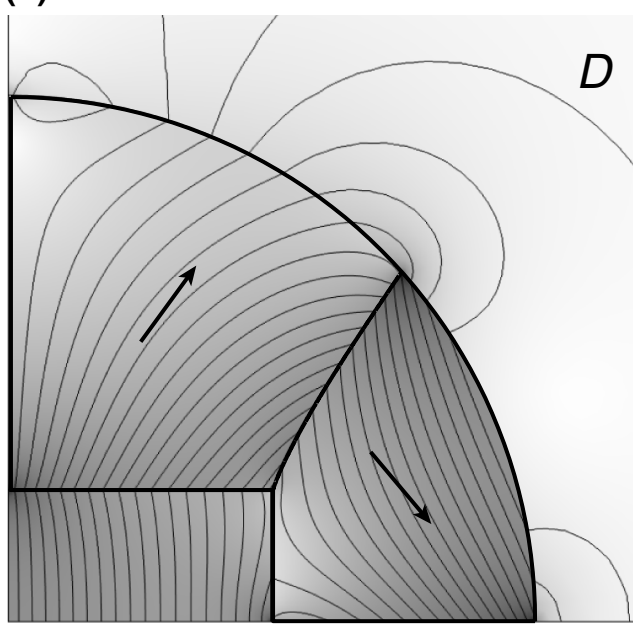

FIG. 4. The steps of the optimization procedure in the configuration space can be symbolically visualized as shown in (a). The horizontal axis corresponds to the directions of the remanence vectors, and the vertical axis corresponds to the subdivision of $R_{m}$ into the regions occupied by the different magnet blocks. The evolution shown in Fig. 3 corresponds to the staircase trajectory $A, B, C, \ldots, D$ shown here, and is analogous to a fixed-point iteration. The procedure terminates at $D$ when both optimality conditions are verified. The flux density generated by the resulting segmented geometry is shown in (b).

Since the magnet domains $\left\{R_{m}^{(k)}\right\}$ and directions of the remanence vectors $\left\{\mathbf{B}_{r 1}^{(k)}\right\}$ are mutually optimal, the solution is at least locally optimal with respect to the whole configuration space (i.e., domains and directions). The corresponding segmentation is shown in Fig. 3(d). The optimization procedure can also be seen as a fixed-point iteration.

One of the strengths of this approach is that it can be applied equivalently to two-dimensional and threedimensional systems. The optimal border between two adjacent segments $k$ and $k^{\prime}$ is still given by points where $\mathbf{H}_{2}$ is equally aligned with the two remanence vectors. For two-dimensional systems this requirement implies that the direction of $\mathbf{H}_{2}$ is constant over the boundary: $\mathbf{H}_{2}$ is equal to the average of the two directions $\mathbf{B}_{r 1}^{(k)}$ and $\mathbf{B}_{r 1}^{\left(k^{\prime}\right)}$. On the other hand, for the three-dimensional case, $\mathbf{H}_{2}$ can have different orientations as long as $\mathbf{H}_{2} \cdot \mathbf{B}_{r 1}^{(k)}=\mathbf{H}_{2} \cdot \mathbf{B}_{r 1}^{\left(k^{\prime}\right)}$. In any case the optimal regions $R_{m}^{(k)}$ are calculated by applying Eq. (11).

\section{Geometrical interpretation}

In both the two-dimensional case and the threedimensional case the problem of optimal segmentation of a given region $R_{m}$ can also be seen from a geometrical point of view.

For the two-dimensional case the segmentation problem is equivalent to maximizing the perimeter of a piecewiselinear approximation of the smooth curve $\mathcal{H}(\psi)$ defined in Eq. (7) in Ref. [10], as also discussed in this work. Each line segment of the approximant corresponds to a magnet block. When the directions of the vectors $\mathbf{B}_{r 1}^{(k)}$ are optimal, the piecewise-linear curve is inscribed in the smooth curve $\mathcal{H}(\psi)$; that is, the two endpoints of each of the line segments lie on $\mathcal{H}(\psi)$. It can also be shown that when the perimeter of the piecewise-linear curve is maximum, the tangent vector to the smooth curve bisects the angle between two consecutive line segments of the piecewiselinear curve. When the latter condition is verified, the regions $R_{m}^{(k)}$ are optimal. The diagrams shown in the topright corner in Fig. 3(a)-3(d) highlight the geometrical interpretation of the two properties.

We now show that the three-dimensional problem is equivalent to the generation of a centroidal Voronoi tessellation $[24,25]$. This special class of a Voronoi diagram has numerous applications, such as data clustering and image compression [24]. A brief introduction to centroidal Voronoi tessellations is given in Appendix A.

It is convenient to express the vector $\mathbf{H}_{2}$ at a point $\mathbf{x}$ in spherical coordinates. Denoting by $H_{2 x}, H_{2 y}$, and $H_{2 z}$ the three components of the vector in Cartesian coordinates, we have

$$
\begin{aligned}
& H_{2 x}(\mathbf{x})=H_{2}(\mathbf{x}) \sin \vartheta(\mathbf{x}) \cos \psi(\mathbf{x}), \\
& H_{2 y}(\mathbf{x})=H_{2}(\mathbf{x}) \sin \vartheta(\mathbf{x}) \sin \psi(\mathbf{x}), \\
& H_{2 x}(\mathbf{x})=H_{2}(\mathbf{x}) \cos \vartheta(\mathbf{x}),
\end{aligned}
$$

where $\mathrm{H}_{2}$ denotes the norm of the virtual field (i.e., $\left.H_{2}=\left\|\mathbf{H}_{2}\right\|\right)$ and $\vartheta$ and $\psi$ denote the inclination and azimuthal angles of the virtual field, respectively. The two angles can be obtained from the Cartesian components using $\vartheta=\arccos \left(H_{2 z} / H_{2}\right)$ and $\psi=\arctan \left(H_{2 y} / H_{2 x}\right)$. It 
is straightforward to generalize the discussion presented in Appendix A in Ref. [10] to prove that if the virtual field has the same directions at two points $\mathbf{x}$ and $\mathbf{x}^{\prime}$, then it is not optimal to assign these two points to different magnet blocks. Two vectors characterized by the same values of $\vartheta$ and $\psi$ are oriented in the same direction, and thus the corresponding points of space belong to the same magnet block. The connectedness of the resulting region is discussed in Sec. IV A. In conclusion, the optimal segmentations of the magnet region $R_{m}$ must be searched for among the possible partitions of the $\psi-\vartheta$ plane into an equal number of regions.

Since we assume a fixed remanence magnitude $B_{r}$, the unknown variables of the problem are the directions of the vectors $\left\{\mathbf{B}_{r 1}^{(k)}\right\}$. We thus consider the set of all the points on a unit sphere of the $\left(H_{2 x}, H_{2 y}, H_{2 z}\right)$ space. We refer to this vector space as the "HII space" and to the unit sphere as the " $\hat{\mathbf{H}}$ sphere." Given a subset $\mathcal{R}$ of the $\hat{\mathbf{H}}$ sphere, we denote by $R$ the corresponding set of the real space; that is, the locus of all points $\mathbf{x}$ where the direction of the virtual field $\mathbf{H}_{2}(\mathbf{x})$ is given by an element of $\mathcal{R}$. The set $R$ is thus defined as

$$
R=\left\{\mathbf{x} \in R_{m}:[\psi(\mathbf{x}), \vartheta(\mathbf{x})] \in \mathcal{R}\right\}
$$

Our problem is to maximize the alignment of the remanence vectors with $\mathbf{H}_{2}$. It is hence natural to consider the formalism of elliptic geometry. In this framework the distance between two vectors $\mathbf{V}$ and $\mathbf{W}$ can be expressed in vector notation as

$$
d_{\mathrm{sph}}(\mathbf{V}, \mathbf{W})=\arccos \left(\frac{\mathbf{V} \cdot \mathbf{W}}{\|\mathbf{V}\|\|\mathbf{W}\|}\right) .
$$

This metric is also referred to as the "great-circle distance" since the geodetics are given by great circles. The set of all points equidistant from two other arbitrary points is also given by a great circle [28]; that is, the intersection of the sphere with a plane passing by the origin.

Let us now consider Eq. (8) defining the functions $L^{(k)}(\mathbf{x})$. The scalar product $\mathbf{B}_{r 1}^{(k)} \cdot \mathbf{H}_{2}(\mathbf{x})$ is proportional to the cosine of the great-circle distance on the unit sphere between the corresponding vectors:

$$
\mathbf{B}_{r 1}^{(k)} \cdot \mathbf{H}_{2}(\mathbf{x})=B_{r} H_{2}(\mathbf{x}) \cos \left[d_{\mathrm{sph}}\left(\mathbf{B}_{r 1}^{(k)}, \mathbf{H}_{2}(\mathbf{x})\right)\right] .
$$

One notices that all the remanence vectors have the same norm $B_{r}$, and that the arccosine function is monotonically decreasing. For these reasons, maximizing the scalar product at the point $\mathbf{x}$ with respect to index $k$ is equivalent to minimizing the spherical distance. This argument illustrates why the maps $\mathcal{R}_{m}^{(k) \text { opt }}$ of the optimal regions can also be seen as the cells of the Voronoi tessellation in spherical geometry generated by the set of directions $\left\{\mathbf{B}_{r 1}^{(k)}\right\}$ :

$$
\begin{aligned}
\mathcal{R}_{m}^{(k) \text { opt }} & =\left\{(\psi, \vartheta) \in \mathcal{R}_{m}: d_{\mathrm{sph}}\left(\mathbf{B}_{r 1}^{(k)}, \hat{\mathbf{e}}_{\psi, \theta}\right)\right. \\
& \left.\leq d_{\mathrm{sph}}\left(\mathbf{B}_{r 1}^{\left(k^{\prime}\right)}, \hat{\mathbf{e}}_{\psi, \theta}\right) \forall k^{\prime} \neq k\right\},
\end{aligned}
$$

where $\hat{\mathbf{e}}_{\psi, \vartheta}$ is the unit vector (i.e., a point on the $\hat{\mathbf{H}}$ sphere) having orientation given by $\psi$ and $\vartheta$. One notices the similarity between Eqs. (18) and (A1) in Appendix A. Figure 5(a) shows an example segmentation of the unit sphere. The directions $\left\{\mathbf{B}_{r 1}^{(k)}\right\}$ generating the segmentation are indicated by the circles lying on the spherical surface. The optimal border between two regions $\mathcal{R}_{m}^{(k) \text { opt }}$ and $\mathcal{R}_{m}^{\left(k^{\prime}\right) \text { opt }}$ is indicated by the dashed line; the border lies on a great circle.

We now construct a scalar density function $\mathcal{H}_{2}(\psi, \vartheta)$ on the $\hat{\mathbf{H}}$ sphere. To do so, we consider the change of variable from the $(x, y, z)$ space to the $\left(H_{2}, \vartheta, \psi\right)$ space. We denote by $g$ the modulus of the determinant of the Jacobian of this transformation. Then we have the following equivalence:

$$
\int_{R} d V \mathbf{H}_{2}(\mathbf{x})=\int_{\mathcal{R}} d \vartheta d \psi \hat{\mathbf{e}}_{\psi, \theta} \int_{0}^{+\infty} d H_{2} g\left(H_{2}, \vartheta, \psi\right) H_{2} .
$$

Denoting by $d S=\sin \vartheta d \vartheta d \psi$ the spherical surface element and defining the density function $\mathcal{H}_{2}(\psi, \vartheta)$ as

$$
\mathcal{H}_{2}(\psi, \vartheta)=\int_{0}^{+\infty} d H_{2} \frac{g\left(H_{2}, \vartheta, \psi\right) H_{2}}{\sin \vartheta}
$$

we can rewrite Eq. (19) as

$$
\int_{R} d V \mathbf{H}_{2}(\mathbf{x})=\int_{\mathcal{R}} d S \mathcal{H}_{2}(\psi, \vartheta) \hat{\mathbf{e}}_{\psi, \theta} .
$$

We now consider the vectors $\mathcal{H}_{2}^{(k)}$, defined in Eq. (6), which determine the optimal directions $\mathbf{B}_{r 1}^{(k) \text { opt }}$. The optimal vectors for the set of regions $R_{m}^{(k)}$ are aligned with the centroids of the corresponding maps $\mathcal{R}_{m}^{(k)}$ on the $\hat{\mathbf{H}}$ sphere:

$$
\mathcal{H}_{2}^{(k)} \propto \frac{\int_{\mathcal{R}_{m}^{(k)}} d S \mathcal{H}_{2}(\psi, \vartheta) \hat{\mathbf{e}}_{\psi, \theta}}{\int_{\mathcal{R}_{m}^{(k)}} d S \mathcal{H}_{2}(\psi, \vartheta)}
$$

The normalization factor has no effect on the results because the spherical distance depends only on the relative orientation between the two vectors. One notices the similarity between Eqs. (22) and (A2) in Appendix A. Figure 5(b) shows an arbitrary (i.e., nonoptimal) partition of the $\hat{\mathbf{H}}$ sphere and the corresponding optimal vectors $\mathbf{B}_{r 1}^{(k) \text { opt }}$. These points are the centroids of the corresponding regions. 
(a)

$$
\begin{aligned}
& \text { Nonoptimal }\left\{\mathbf{B}_{r 1}^{(k)}\right\} \\
& \text { and their optimal }\left\{\mathcal{R}_{m}^{(k)}\right\} \\
& \text { (Voronoi cells) }
\end{aligned}
$$

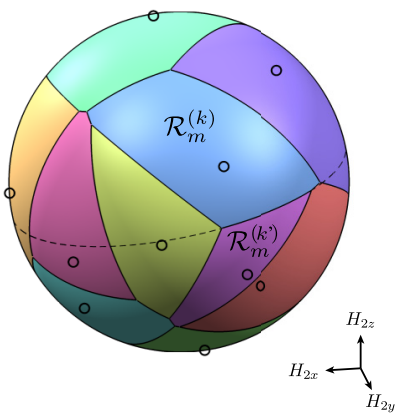

(b)

$$
\begin{aligned}
& \text { Nonoptimal }\left\{\mathcal{R}_{m}^{(k)}\right\} \\
& \text { and their optimal }\left\{\mathbf{B}_{r 1}^{(k)}\right\} \\
& \text { (centroids) }
\end{aligned}
$$

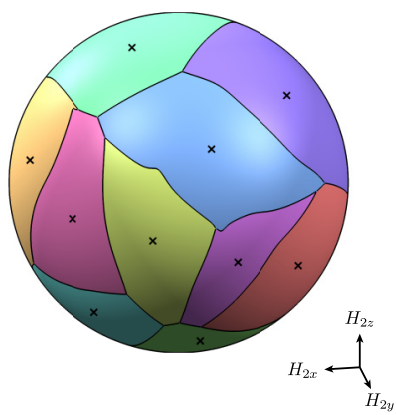

(c)

Mutually optimal

$\left\{\mathbf{B}_{r 1}^{(k)}\right\}$ and $\left\{\mathcal{R}_{m}^{(k)}\right\}$

(centroidal Voronoi diagram)

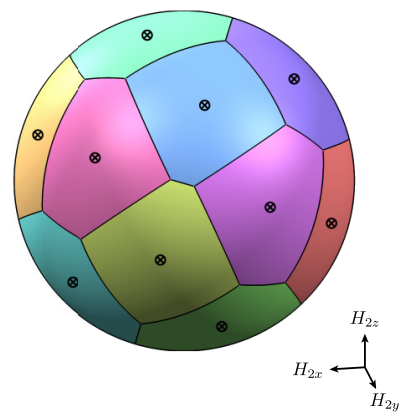

FIG. 5. The properties of optimal segmentations can be geometrically interpreted by considering the $\hat{\mathbf{H}}$ sphere; that is, the set of normalized $\mathbf{H}_{2}$ vectors. (a) an arbitrary set of directions $\left\{\mathbf{B}_{r 1}^{(k)}\right\}$ indicated by the open circles. The corresponding globally optimal regions $\left\{\mathcal{R}_{m}^{(k) \text { opt }}\right\}$ are the Voronoi cells generated by the directions. The optimal border between two adjacent regions is thus a great circle. The optimal border between $\mathcal{R}_{m}^{(k) \text { opt }}$ and $\mathcal{R}_{m}^{\left(k^{\prime}\right) \text { opt }}$ is indicated by the dashed line. (b) An arbitrary subdivision of the $\hat{\mathbf{H}}$ sphere into nonoptimal regions $\left\{\mathcal{R}_{m}^{(k)}\right\}$. The corresponding globally optimal directions $\left\{\mathbf{B}_{r 1}^{(k) \text { opt }}\right\}$, which are the centroids of the corresponding regions, are indicated by the crosses. (c) A locally optimal segmentation, corresponding to a centroidal Voronoi tessellation on the $\hat{\mathbf{H}}$ sphere. Regions and directions are mutually globally optimal.

When both optimality conditions are simultaneously fulfilled, the segmentation is equivalent to a special class of Voronoi tessellation referred to as "centroidal Voronoi tessellation" (see Appendix A). Figure 5(c) shows an example of such tessellation on the $\hat{\mathbf{H}}$ sphere. The directions and the regions are mutually optimal. In the context of centroidal Voronoi tessellations, the optimization procedure described in Sec. II C is known as Lloyd's method $[24,25,27]$.

Let us consider the three-dimensional example shown in Fig. 6(a). For this example the goal is to create a uniform field oriented along the $x$ direction. The objective is thus to maximize the $x$ component of $\mathbf{H}_{1}$ averaged over the cubic air gap labeled as $R_{g}$. The design area $R_{m}$ is chosen as a block of the same height as the air gap and with double the width and depth. Figure 6(a) shows the virtual system: the optimization procedure is applied to one quarter of the geometry $(x>0$ and $y>0)$ and then extrapolated to the other quarters by our considering the symmetry of this problem. The density function $\mathcal{H}(\varphi, \vartheta)$ is calculated from the virtual field distribution $\mathbf{H}_{2}(\mathbf{x})$ by our applying Eq. (20). (a) Virtual system

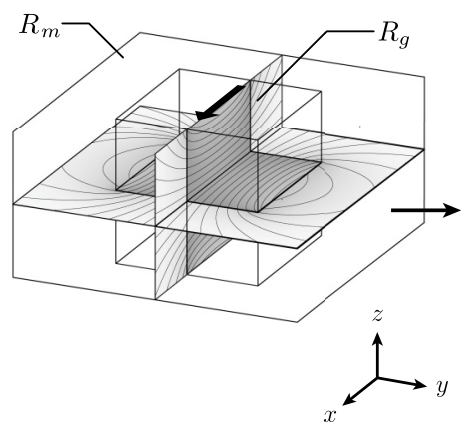

(b)

Optimization procedure

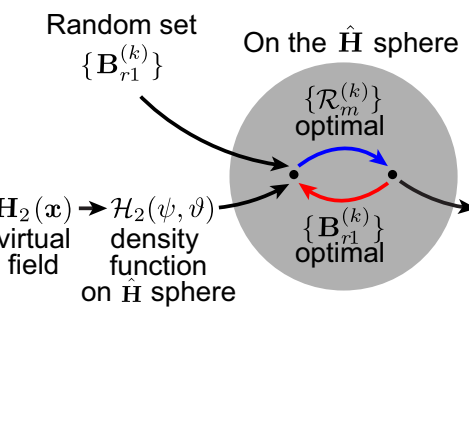

(c) Real system (optimally segmented)

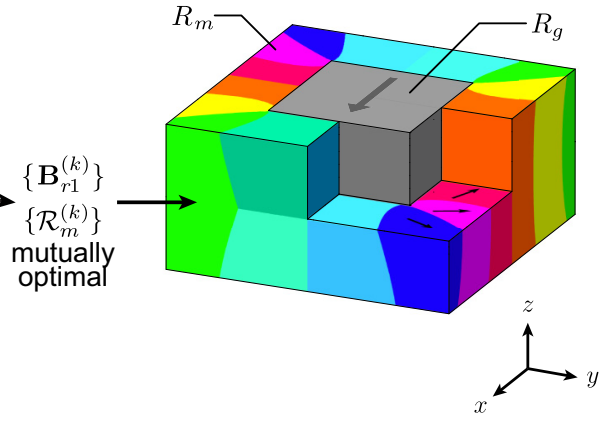

FIG. 6. (a) Virtual system for the example problem of maximizing the $x$ component of the field averaged over a cubic air gap. The field lines of $\mathbf{B}_{2}$ are shown; the norm of $\mathbf{B}_{2}$ is indicated by the grayscale, with darker shades corresponding to larger field magnitude. (b) The optimization procedure on the $\hat{\mathbf{H}}$ sphere. The density function $\mathcal{H}_{2}(\varphi, \vartheta)$ is calculated from the virtual field $\mathbf{H}_{2}$. Lloyd's iteration is applied from a set of random directions until a configuration for which regions and directions are mutually optimal is reached. (c) Optimally segmented real system obtained from the optimization procedure. The different blocks are indicated by different colors and the corresponding optimal remanence directions are indicated by the black arrows. 

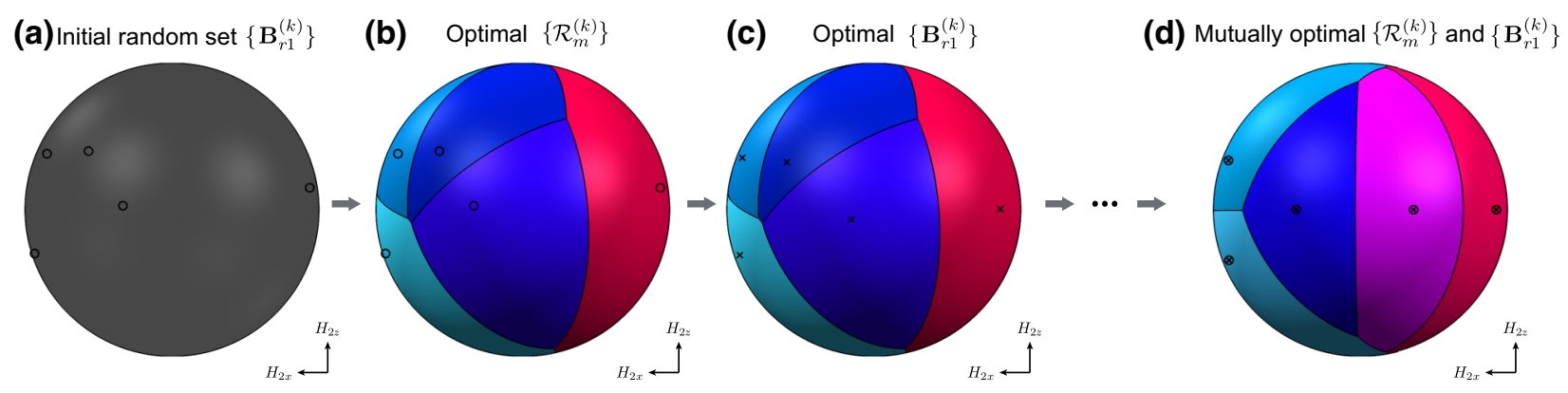

FIG. 7. The optimization procedure for the problem in Fig. 6: Lloyd's algorithm applied to the $\hat{\mathbf{H}}$ sphere. (a) Set of five random initial directions, indicated by the open circles. (b) Corresponding optimal partition into Voronoi cells having those directions as generators. (c) The directions are updated on the basis of the regions: each direction becomes the centroid of its cell, indicated by the crosses. The partition into regions is updated again on the basis of the new directions. This iterative procedure is continued until the centroidal Voronoi tessellation shown in (d) is obtained. For this configuration, corresponding to the segmentation in Fig. 6(c), regions and directions are mutually optimal.

As illustrated in Fig. 6(b), the iterative optimization procedure discussed in Sec. IIC can also be applied to three-dimensional problems by working on the $\hat{\mathbf{H}}$ sphere. The starting step is to randomly generate a set of initial directions $\left\{\mathbf{B}_{r 1}^{(k)}\right\}$. The optimal regions $\left\{\mathcal{R}_{m}^{(k) \text { opt }}\right\}$ for this initial set can then easily be calculated, as they are the Voronoi cells generated by the directions. The directions are thus updated on the basis of the regions: the new directions are the centroids calculated from the partition and from the density function $\mathcal{H}_{2}(\varphi, \vartheta)$. The procedure continues by alternately optimizing regions and directions until a configuration with mutually optimal regions and directions is found. When the optimization procedure is completed, the real-space regions $\left\{R_{m}^{(k) \text { opt }}\right\}$ are calculated from the corresponding maps $\left\{\mathcal{R}_{m}^{(k) \text { opt }}\right\}$ on the $\hat{\mathbf{H}}$ sphere by applying Eq. (15).

Figure 6(c) shows a cutaway diagram of the optimally segmented real system. Each quarter of the geometry is segmented with $N=5$ segments, for a total of 20 segments.

Some steps of the optimization procedure on the $\hat{\mathbf{H}}$ sphere are shown in Fig. 7. The starting step is to randomly generate $N=5$ starting directions, as shown in Fig. 7(a). The spherical Voronoi tessellation generated by the initial set of directions can be calculated from Eq. (18) and is shown in Fig. 7(b). Subsequently the set of directions are updated again on the basis of Eq. (22). These directions are thus the centroids of the regions calculated in the previous step of the iteration. The iteration is continued until a centroidal Voronoi tessellation is reached. The final configuration is shown in Fig. 7(d) and corresponds to the segmentation shown in Fig. 6(c).

\section{E. Optimal magnet-air border}

So far we have considered segmentations for which the magnet region $R_{m}$ is filled by the $N$ permanent-magnet blocks. We now examine the more-general problem of determining the optimal external border of the blocks; that is, the border between each block and the surrounding air. Since the permeability field $\underline{\underline{\mu}}(\mathbf{x})$ is assumed to be predetermined and it affects the virtual field distribution $\mathbf{H}_{2}(\mathbf{x})$, the magnet-air border can be determined only if the relative permeability of the permanent magnet is close to 1 . This approximation is not an unrealistic description of the behavior of widely used permanent-magnet materials [29]. In other parts of the magnetic system, the permeability may be different from 1 .

In the continuous limit discussed in Sec. II A the optimal remanence distribution is a field of norm $B_{r}$ aligned at any point with the virtual field $\mathbf{H}_{2}$. In this limit, $\mathcal{S}$ is thus given by:

$$
\mathcal{S}_{\infty}=\int_{R_{m}} d V L_{\infty}(\mathbf{x})
$$

where the scalar field $L_{\infty}(\mathbf{x})$ expresses the contribution to $\mathcal{S}_{\infty}$ from a point $\mathbf{x}$, and is defined as

$$
L_{\infty}(\mathbf{x})=B_{r}\left\|\mathbf{H}_{2}(\mathbf{x})\right\| .
$$

As discussed in Sec. B in Ref. [10], in this limit the optimal external border of the permanent magnet is a level curve of $L_{\infty}(\mathbf{x})$ : it is more convenient to use permanentmagnet material at points with a higher value of $L_{\infty}$. The value $L_{0}$ that the field $L_{\infty}$ assumes on the external border can be determined to satisfy a given constraint on the total volume $V_{m}$ of permanent-magnet material. A smaller threshold value $L_{0}$ gives a larger magnet volume $V_{m}$.

Applying the same reasoning to a segmented permanent magnet, we notice that the external magnet-air border of the $k$ th uniformly magnetized block is given by a level curve of the scalar field $L^{(k)}(\mathbf{x})$ defined in Eq. (8). We denote by $L_{0}^{(k)}$ the value of $L^{(k)}(\mathbf{x})$ on the external border of 
(a)

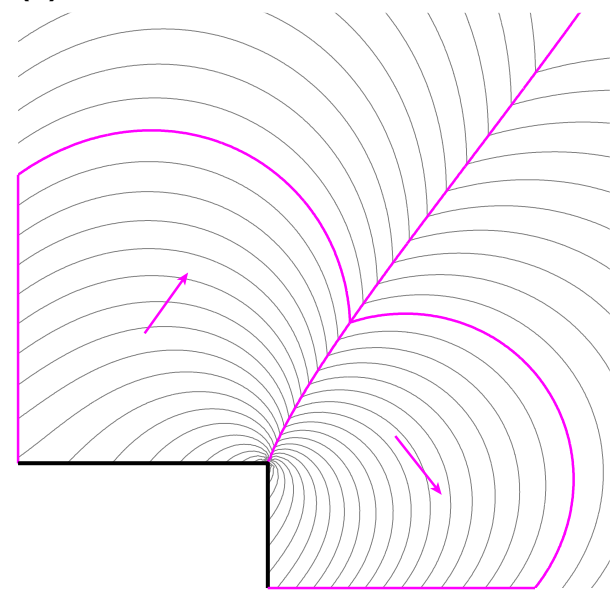

(b)

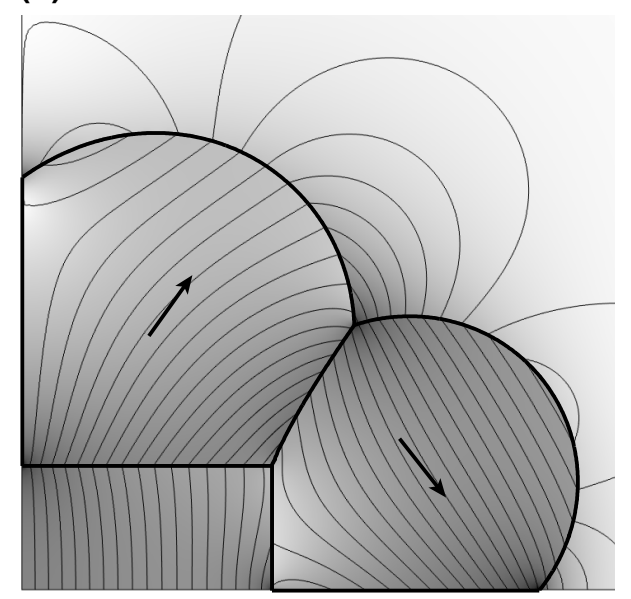

FIG. 8. The optimal external border between each magnet block and the surrounding air is calculated for the same optimization problem shown in Figs. 2, 3, and 4(b). The level curves of the scalar fields $L^{(k)}(\mathbf{x})$ are shown as thin lines in (a). The thick magenta lines are the final external borders satisfying a given arbitrary constraint on the magnet volume. The resulting fluxdensity distribution $\mathbf{B}_{1}$ is shown in (b). the block $k$. If $L_{0}^{\left(k^{\prime}\right)}<L_{0}^{(k)}$ for some $k^{\prime} \neq k$, then it would be convenient to reassign the part of $R_{m}^{\left(k^{\prime}\right)}$ with lower value of $L^{\left(k^{\prime}\right)}(\mathbf{x})$ to the block $k$. This argument implies that all the fields $L^{(k)}(\mathbf{x})$ assume the same value $L_{0}$ on the external border.

The iterative procedure described in the previous section can be generalized so that the external magnet-air borders of all the blocks are also optimal. Whenever the optimal set of regions $R_{m}^{(k) \text { opt }}$ are recomputed on the basis of a fixed set of remanence vectors, the new value of $L_{0}$ satisfying a given constraint on the total volume $V_{m}$ is recalculated and the external borders of all the domains are determined accordingly.

Let us consider the same two-dimensional example examined in the previous sections. In this case instead of filling the region $R_{m}$ enclosed by an external circle, we calculate the optimal external border leading to the same total magnet volume $V_{m}$. The level curves of $L^{(k)}(\mathbf{x})$ are shown in Fig. 8(a) as thin black lines: the curves for $L^{(1)}(\mathbf{x})$ are plotted only inside $R_{m}^{(1)}$, and similarly for $L^{(2)}(\mathbf{x})$. The optimal external borders extend further away from the air gap in the central region of the segments; that is, where the virtual field $\mathbf{H}_{2}$ has better alignment with the remanence vectors $\mathbf{B}_{r 1}^{(k)}$.

The configuration shown in Fig. 8(a) corresponds to the final solution of the iterative procedure. The level curves corresponding to the value $L_{0}$ that complies with the volume constraint are the optimal external borders of the two magnet blocks. Since on the optimal border between two adjacent segments $\mathbf{H}_{2}$ is equally aligned with the two remanence vectors, the magnet-air borders of the two blocks intersect the magnet-magnet border at the same point. The real magnetic flux density $\mathbf{B}_{1}$ for this solution is shown in Fig. 8(b) with the same notation as used in Figs. 2 and 4(b).

The geometry shown in Fig. 1 is an example of threedimensional system with an optimal external interface between each segment and the surrounding air. As for the two-dimensional case, the external border of each segment extends further away from $R_{g}$ in the region where the alignment between the remanence vector and the virtual field is maximum.

\section{F. Implementation}

The first step of our numerical implementation of the optimization procedure is to use finite-element methods to calculate the virtual field distribution $\mathbf{H}_{2}(\mathbf{x})$ corresponding to the given objective functional $\mathcal{S}$ and permeability distribution $\underline{\underline{\mu}}(\mathbf{x})$. For this purpose we use COMSOL MULTIPHYSICS, although when no soft magnetic materials are present it would be possible to compute the virtual field with analytical techniques. The virtual field $\mathbf{H}_{2}$ is then imported into a MATLAB environment and the density function $\mathcal{H}_{2}(\psi, \theta)$ defined in Eq. (20) is calculated from $\mathbf{H}_{2}$. The density function $\mathcal{H}_{2}$ is thus stored for later use as a MATLAB-formatted binary file.

The second step is to use MATLAB to perform the iterative procedure described in Sec. II C, or the generalization described in Sec. II E. The starting point is a set of $N$ remanence directions randomly chosen among the directions that $\mathbf{H}_{2}$ assumes in $R_{m}$. The iterative procedure is stopped when the remanence vectors have converged. In practice this criterion is implemented by requiring that the variation between two consecutive iterations is less than a certain tolerance threshold. The mathematical style pseudocode corresponding to the optimization iterative procedure is provided in Ref. [30].

It is important to stress that the first step does not need to be repeated to perform the optimization with a different magnet volume or number of segments. As a result of the optimization procedure, the value of $\mathcal{S}$ is automatically calculated. It is also straightforward to calculate the reduction of $\mathcal{S}$ with respect to the value $\mathcal{S}_{\infty}$ corresponding to the continuous case $N \rightarrow \infty$. The calculations of $\mathcal{S}$ and $\mathcal{S}_{\infty}$ do not require the third step described below. 
(a)

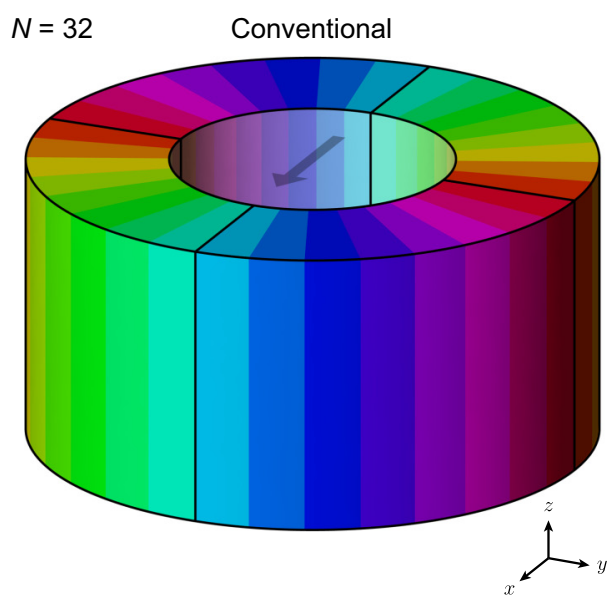

(b)

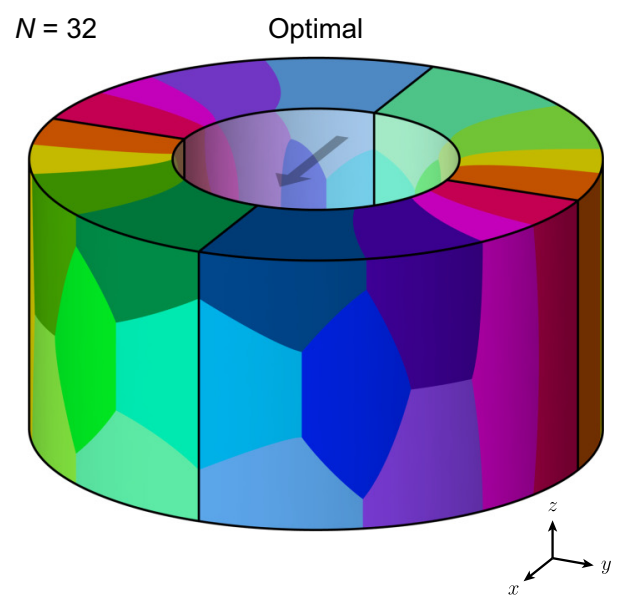

FIG. 9. Halbach-cylinder geometry, with cylindrical design region $R_{m}$. (a) Conventional segmentation of the magnet region using eight segments per quadrant (i.e., $N=32$ segments in total). (b) Optimized segmentation having the same number of segments.
To compute the real field distribution $\mathbf{H}_{1}(\mathbf{x})$ generated by the optimal configuration, we need to perform an optional third step: the optimal solution is imported into COMSOL MULTIPHYSICS, which is then used to calculate the real field distribution. In our implementation the third step requires the largest fraction of the total computation time.

Our implementation of the optimization framework presented in this paper is publicly available in Ref. [26].

\section{EXAMPLES}

We have demonstrated our approach on a number of magnet-design problems, which are examined in this section. The framework of our optimization approach assumes linear constitutive relations: the permanent magnet has relative permeability $\mu_{r}=1$ and the iron parts have relative permeability $\mu_{r}=1000$. The validity of these assumptions is discussed in Sec. IV A. Because of the linearity of the constitutive relation and the objective functional $\mathcal{S}$, the results scale linearly with the norm of the remanence $B_{r}$. For this reason, we normalize the results by assuming remanence $B_{r}=1 \mathrm{~T}$.

We express the performance in terms of the value of $\mathcal{S}\left[\mu_{0} \mathbf{H}_{1}\right]$ normalized by the air-gap integral of the squared norm of $\mathbf{u}$. This normalized linear functional is denoted by $\beta$. When $\mathbf{u}$ is a normalized uniform field (e.g., oriented in the $x$ direction), $\beta$ is equal to the average of the $x$ component of the flux density. In other cases $\beta$ is a generalized figure of merit of field intensity proportional to $\mathcal{S}$, which is expressed in teslas when $\mathbf{u}$ is nondimensional.

We also wish to quantify the quality of the field with respect to the desired distribution $\mathbf{u}(\mathbf{x})$. For this purpose we introduce a nonlinear functional $\gamma^{2}$. This functional, defined in Appendix B, is physically interpreted as the fraction of air-gap magnetic energy stored in the component of the field distribution that is proportional to $\mathbf{u}(\mathbf{x})$. The remaining fraction of energy is stored in the irrelevant component of the field. The functional $\gamma^{2}$ is normalized in such a way that when $\mathbf{H}_{1}(\mathbf{x})$ is proportional to $\mathbf{u}(\mathbf{x}), \gamma^{2}$ is equal to 1 . For this reason, the value of $\gamma^{2}$ can be expressed as a percentage.

\section{A. Transverse field in a cylindrical cavity Halbach array}

As a first example, we consider the optimal threedimensional segmentation of a finite-length Halbach cylinder [16]. An example is shown in Fig. 9. The objective is to maximize the $x$ component of the field averaged over the central cylindrical air gap. The radius $r_{I}$ of the air gap is half of its height $\ell_{I}$ along the vertical $z$ direction: $\ell_{I}=2 r_{I}$. With this choice of aspect ratio, the end effects are not negligible, but are not as intense as to render the standard Halbach-cylinder geometry completely nonoptimal. The external radius $r_{O}$ of the magnet-design region is twice the inner radius: $r_{O}=2 r_{I}$.

For the results presented in this section the optimization procedure is applied to the first quadrant of the $x-y$ plane, and the other three quadrants are obtained by our taking advantage of the mirror symmetries across the planes $x=0$ and $y=0$. We consider different alternative magnetdesign regions $R_{m}$, all corresponding to the same total magnet volume $V_{m}=\pi \ell_{I}\left(r_{O}^{2}-r_{I}^{2}\right)$ :

(a) Cylindrical region, as the one shown in Fig. 9(b).

(b) Spherical shell [31], open on the top and bottom, as shown in Fig. 10. If we denote by $r_{S}$ the external radius of the spherical shell, the magnet volume $V_{m}=(4 \pi / 3)\left(r_{S}^{2}-\right.$ $\left.r_{I}^{2}\right)\left(1-r_{I}^{2} / r_{S}^{2}\right)^{1 / 2}$. To maintain the same volume, the radius $r_{S} \approx 1.93 \times r_{I}$.

(c) Optimal external border [32], as shown in Fig. 11. The external border of each segment is calculated with the procedure described in Sec. II E.

The results of our optimization approach are compared with the results of conventional segmentation (i.e., vertical slices) of a Halbach cylinder having the same design 

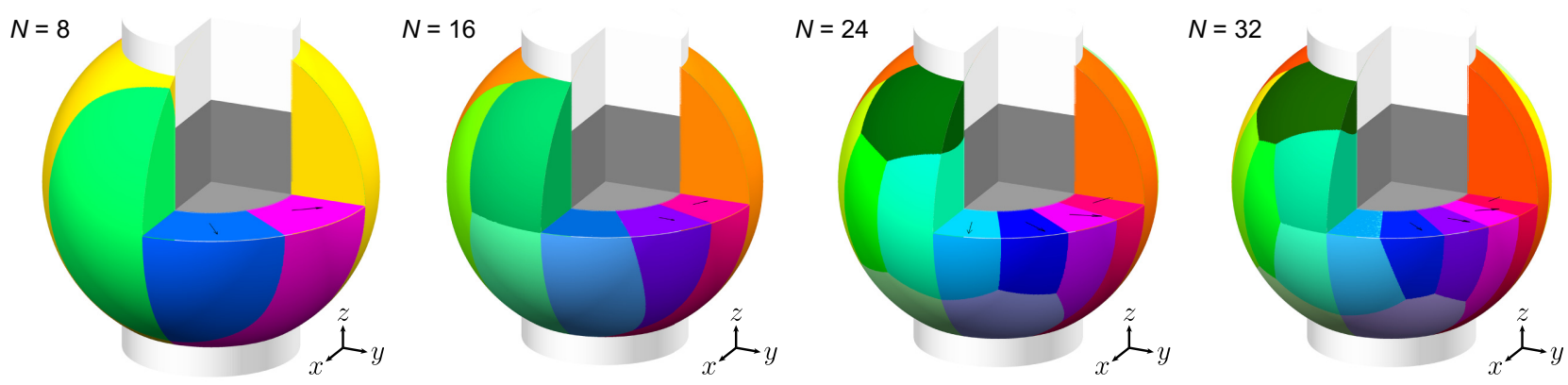

FIG. 10. Segmentation of the first quadrant of the $x-y$ plane of the magnetic domain for the optimization problem shown in Fig. 9(b). Each plot from left to right corresponds to a different number of segments in increasing order: $N=8,16,24,32$.

region as the one in Fig. 9(b). An example of conventional segmentation is shown in Fig. 9(a).

It is worth noticing that the spherical shell is extremely close to the optimal magnet region for the continuous limit $N \rightarrow \infty$ : as explained in Sec. II E, when the relative permeability of the permanent magnet is $\mu_{r}=1$, the optimal border of the magnet region is given by one of the level surfaces of $B_{r}\left\|\mathbf{H}_{2}\right\|$, which at sufficient distance from $R_{g}$ become nearly exactly spherical.

The results for the four cases are plotted in Fig. 12(a) as a function of the total number of segments $N$ : the top panel shows the values of $\beta \propto \mathcal{S}$, expressed in teslas, and the bottom panel shows the values of $\gamma^{2}$ expressed as a percentage.

The conventional Halbach cylinder shows a lower value of $\beta$ than the other three cases optimized with our approach. The spherical magnet domain is superior to the optimized cylindrical domain except for $N=8$. As expected, the highest value of $\beta$ for each $N$ is always given by the configurations where the optimal external border of each magnet block is optimal (i.e., the blue crosses). In each of the four configurations, $\beta$ generally increases with $N$ and always converges to the continuous limit as $N \rightarrow+\infty$. In this limit the conventional Halbach segmentation $\mathcal{S}$ converges to a lower value than the optimized segmentation of the same magnet region. While for the optimized segmentation the limit is given by Eq. (23), since the conventional segmentation considers only subdivisions along the azimuthal direction, the vertical $z$ component of $\mathbf{H}_{2}$ must not be considered when the limit is being calculated.

We now discuss the quality of the field distribution as quantified by the value of $\gamma^{2}$. As can be seen from the bottom panel in Fig. 12(a), in all cases $\gamma^{2}$ converges to a plateau as $N \rightarrow \infty$. The optimized segmentation over the cylindrical domain gives the worst results in terms of field uniformity. For large $N$ the conventional segmentation gives a slightly better field quality than the spherical domain, while for $N=8$ and $N=16$ the spherical domain performs better. In all cases except for the conventional segmentation, $\gamma^{2}$ decreases as $N$ increases; as more and more magnetic energy is being stored in the air gap, the amount associated with desired field component is increasing, but so is the amount associated with the irrelevant components. Since the ratio between the two quantities gets worse, the value of $\gamma^{2}$ is decreasing. A more generalized discussion of the trade-off between field quality and intensity is presented in Sec. IV A.

The value of $\gamma^{2}$ for all the optimized segmentations oscillates depending on whether $N / 4$ is even or odd. Even
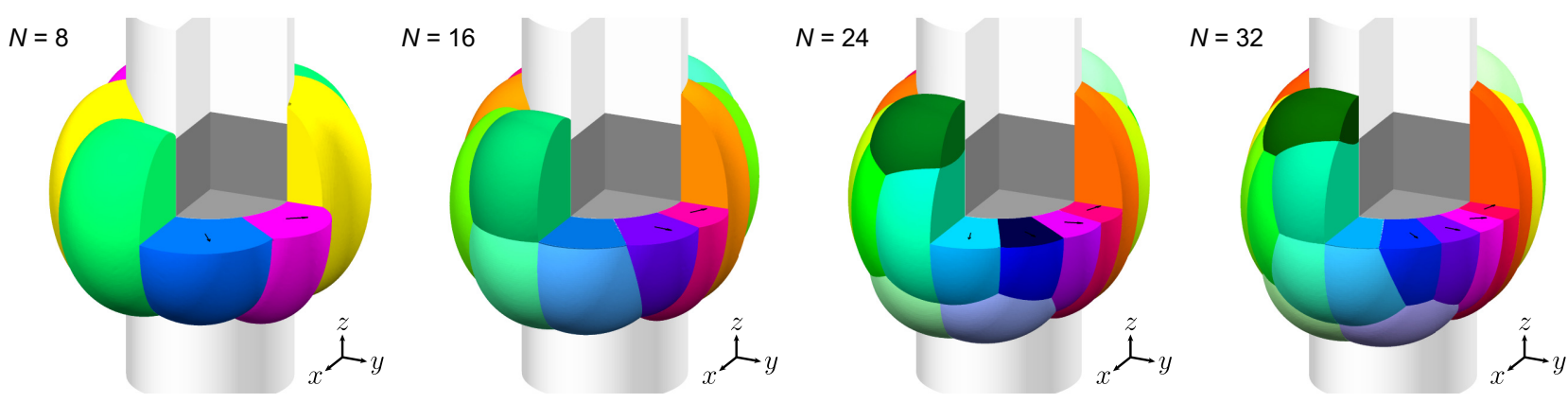

FIG. 11. The same configurations as the ones shown in Fig. 10 but with an optimal external border of each magnet block. The total permanent-magnet volume $V_{m}$ is the same in both cases. 
(a)
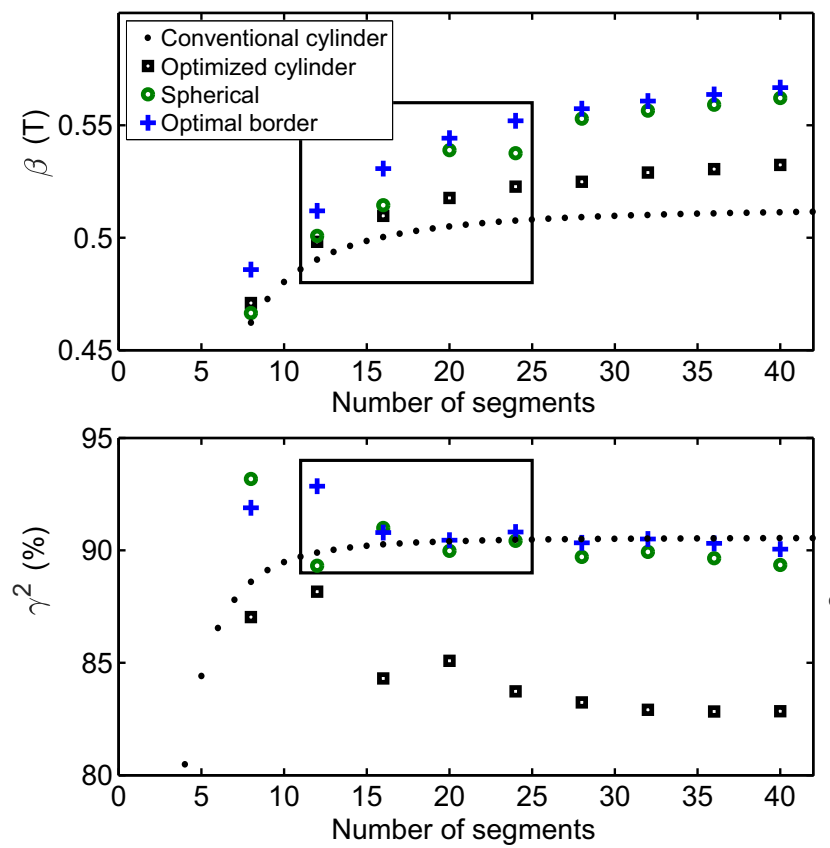

(b)

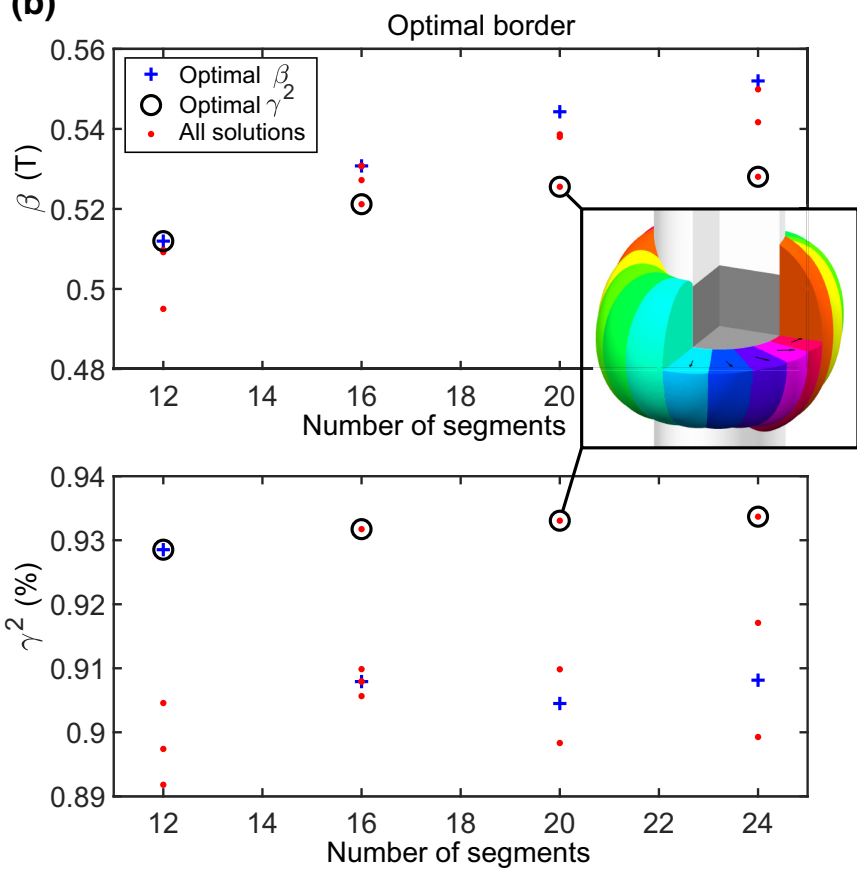

FIG. 12. (a) Results of the optimization problem described in Sec. III A. The purpose is to maximize the $x$ component of the field averaged over a cylindrical air gap $R_{g}$. Different magnet-design regions $R_{m}$ correspond to different markers, as indicated in the key. The conventional Halbach segmentation of the cylindrical domain is shown by the black dots. The top panel shows the values of $\beta$, whereas the bottom panel shows the values of $\gamma^{2}$. In all cases we notice convergence to a plateau in the limit $N \rightarrow \infty$. (b) The various locally optimal solutions for the case of the optimized magnet region, corresponding to the crosses in (a). The solutions giving the maximum value of $\gamma^{2}$ are indicated by the black circles, and are not always optimal for $\beta$, as in the case shown in the inset.

though it is less pronounced, a similar trend is exhibited by $\beta$ for the case of the spherical domain. The cause of this oscillation is a change in the topology of the optimal solution; that is, the solution corresponding to the maximum value of $\beta$. As elaborated on in Sec. IV $\mathrm{B}$, in many cases there are several topologically distinct locally optimal solutions satisfying both optimality conditions discussed before. The number of locally optimal points increases with $N$; for the range of values considered here, there are only a few local optima. As discussed in Sec. IV B, it is very plausible that the segmentations calculated with our approach are the globally maximal solution to the linear optimization problem.

Figure 12(b) shows all the local solutions we find for the case of the optimal external border. The axes of the two panels correspond to the black rectangles in Fig. 12(a). The solution giving the maximum value of $\beta$ are indicated by the crosses, and the solutions giving the maximum value of $\gamma^{2}$ are indicated by the black circles. The remaining local optima are indicated as red dots, and the corresponding geometries are shown in Ref. [30]. As can be seen, for $N=12$ the solution giving the maximum $\beta$ also gives the the maximum $\gamma^{2}$. This coincidence is not verified for $N=16,20$, and 24 , which is not surprising since there is no theoretical reason to expect that $\gamma^{2}$ is maximized by the same configurations that maximize $\beta$. Among the cases encountered during our investigations, the solutions that maximize $\gamma^{2}$ are always given by "Halbach-style" segmentations as the one shown in the inset, or the one in the leftmost panel in Fig. 11 (i.e., $N=8$ ).

\section{B. Finite-length quadrupole magnet}

As a second example, we consider a magnetic system designed to generate a quadrupole field inside a cylindrical cavity. For this example the vertical extension of the air gap is 4 times the inner radius: $\ell_{I}=4 \times r_{I}$. As pointed out in Ref. [33], when one is considering finite-length quadrupoles, the desired air-gap field distribution $\mathbf{u}(\mathbf{x})$ should be

$$
\begin{aligned}
u_{x}(x, y, z)= & \sin x \cos x /\left(\cos ^{2} x+\sinh ^{2} z\right), \\
u_{y}(x, y, z)= & -\sin y \cos y /\left(\cos ^{2} y+\sinh ^{2} z\right), \\
u_{z}(x, y, z)= & -\sinh 2 z /(\cos 2 x+\cosh 2 z) \\
& +\sinh 2 z /(\cos 2 y+\cosh 2 z) .
\end{aligned}
$$

The norm of $\mathbf{u}$ decays as the modulus of $z$ increases. In a similar manner as for the spherical magnet domain 
(a)
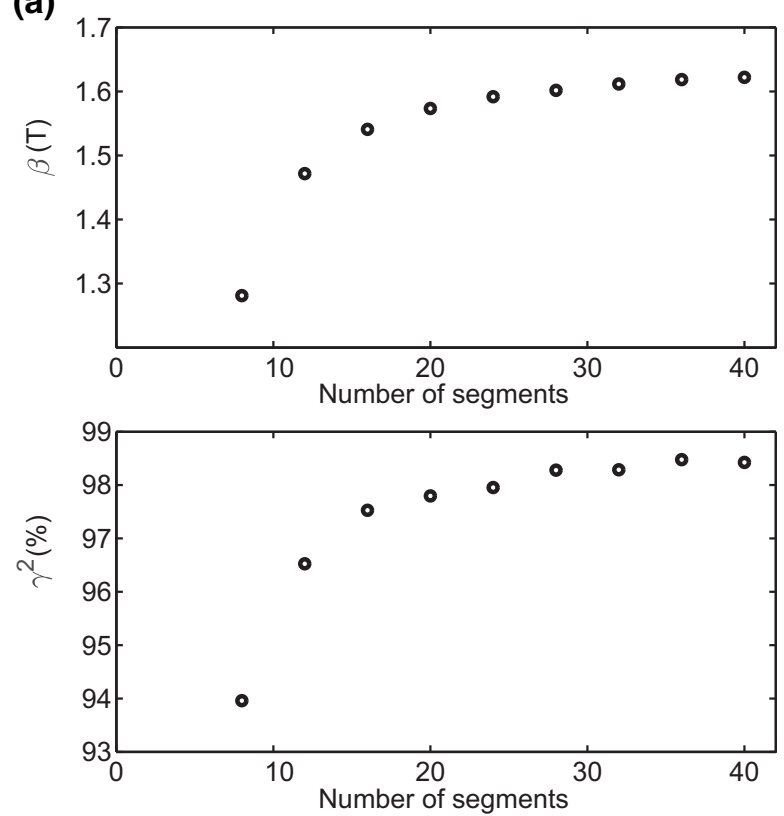

(b)

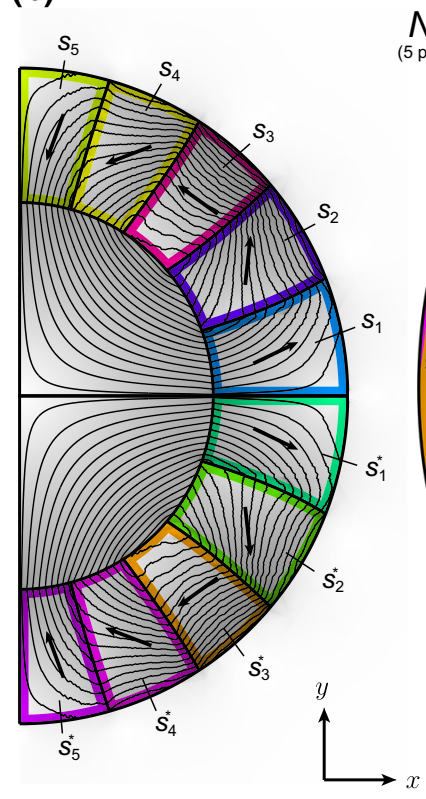

$N=20$

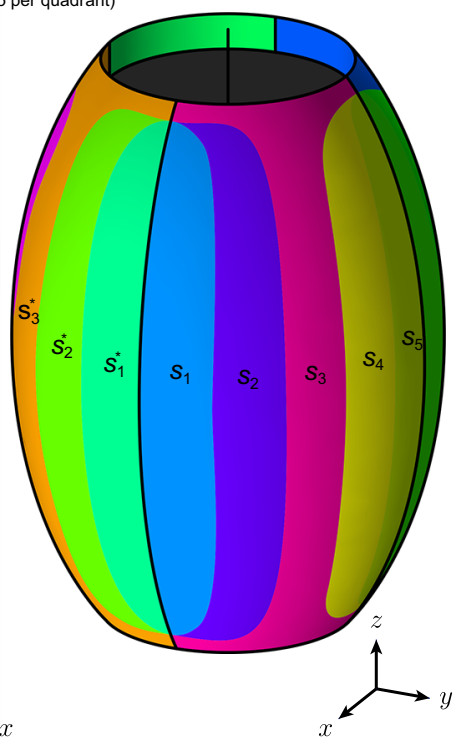

FIG. 13. Magnetic system designed to produce a quadrupole field inside a cylindrical air gap. (a) The results as functions of the total number of segments $N$. The top panel shows the field-intensity figure of merit $\beta$. The bottom panel shows the field-quality figure of merit $\gamma^{2}$. (b) One example of optimized segmentation with $N=20$ total magnet blocks. The cross section of the half-plane $z=0$, $x \geq 0$ shows the field lines of the magnetic flux density $\mathbf{B}_{1}$. The norm of the flux density is indicated by the gray background, with darker shades for higher values. For each segment, the optimal remanence vector is indicated by a black arrow and the color of the border is the same as in the three-dimensional illustration.

discussed in the previous section, we consider an approximation of the optimal magnet region. When the permanentmagnet relative permeability is close to 1 , the optimal region for the continuous case, $N \rightarrow \infty$, is given by a level surface of the norm $\left\|\mathbf{H}_{2}\right\|$ of the virtual field. For the example discussed here, with an arbitrarily chosen constraint on the total magnet volume, the optimal surface is well approximated by a prolate ellipsoid of revolution oriented along the $z$ axis. The radii along the $x$ and $y$ directions, $r_{\perp}$, are approximately $1.69 r_{I}$, and the radius along the $z$ direction, $r_{Z}$, is approximately $2.85 r_{I}$. The cylindrical regions above and below $R_{g}$ are left open to access the inner cavity. The geometry of the system is shown in Fig. 13(b).

We perform the optimization procedure on quarter of the geometry, corresponding to the first quadrant of the $x-y$ plane. The results are shown in Fig. 13(a) as function of the total number of segments $N$. The top panel shows the value of $\beta$ and the the bottom panel shows the value of $\gamma^{2}$. The field precision is considerably better than for the case discussed in the previous section. As expected, $\beta$ and $\gamma^{2}$ increase and converge to a plateau as $N \rightarrow \infty$.

The optimized segmented geometry shown in Fig. 13(b) corresponds to five segments for each quarter (i.e., $N=20$ total segments). As can be seen, for this solution all segments are symmetric with respect to a reflection across the $x-y$ plane. This implies that the $z$ component of the optimal remanence vector is zero for all segments. A cross section corresponding to the half-plane $z=0, x \geq 0$ is shown in the same panel. The field lines of the magnetic flux density $\mathbf{B}_{1}$ are shown as black lines, and the optimal remanence vectors are shown as black arrows. The norm of the flux density is indicated by the shades of gray, with darker shade corresponding to higher field intensity. The border of each segment is highlighted with the same color as in the three-dimensional illustration. As also shown by the corresponding data points in the bottom panel, the air-gap field distribution matches quite precisely the desired distribution $\mathbf{u}(\mathbf{x})$.

\section{Magnetic refrigeration}

In this section we present a rotary device for roomtemperature magnetic refrigeration. The design of this device is shown in Fig. 14(b), and is inspired by a previously published design [34] composed of two coaxial cylinders. The air gap between the two cylinders is filled by vertical regenerators enclosing the magnetocaloric material. Vertically, the regenerators occupy the region of the air gap delimited by the dashed lines shown in Fig. 14(b), corresponding also to the top and bottom faces of the white regions labeled as "high field." The external cylinder 
(a)
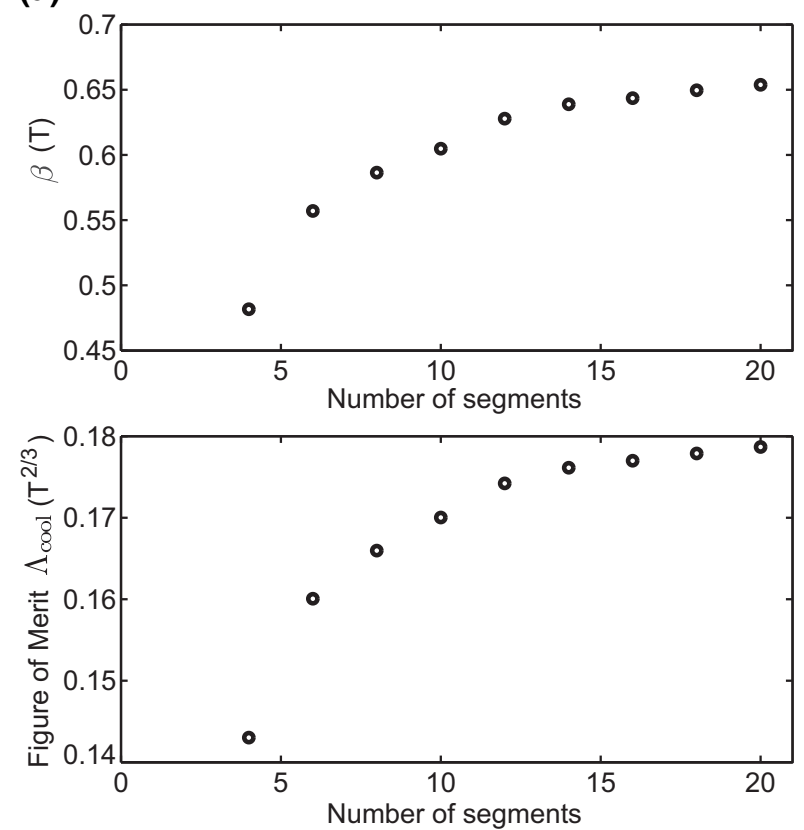

(b)

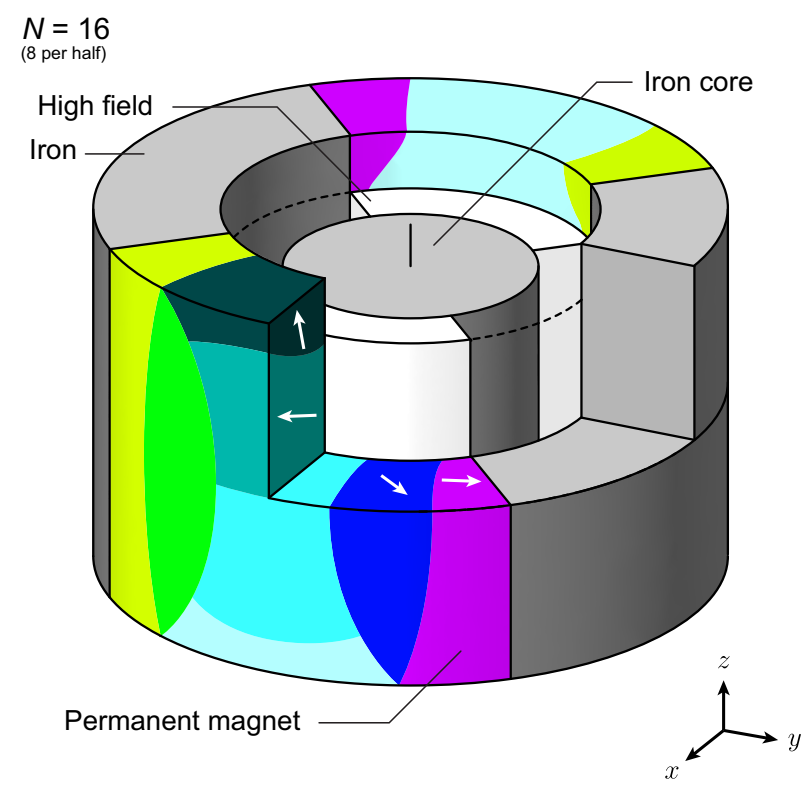

FIG. 14. Results of the optimization procedure applied to the design of a rotary device for room-temperature magnetic refrigeration. (a) Results plotted as a function of the total number of segments $N$. The top panel shows the value of $\beta$, whereas the bottom panel shows the value of the figure of merit $\Lambda_{\text {cool }}$. The values of $\Lambda_{\text {cool }}$ are comparable to those for published designs of magnetic refrigeration devices. (b) Optimized segmented geometry corresponding to eight segments for each half (i.e., $N=16$ segments in total).

incorporates the permanent-magnet blocks, symmetrically placed on opposite sides of the $y-z$ plane, and two iron parts, symmetric with respect to the $x-z$ plane. The internal cylinder is an iron core that closes the magnetic circuit. As the external cylinder rotates, each regenerator experiences a time-varying magnetic field, which alternates between high-field and low-field states.

As an objective vector field $\mathbf{u}(\mathbf{x})$ we define a radial uniform field that is nonzero only in the angular sectors of the air gap corresponding to the white areas shown in Fig. 14(b). Each of these sectors spans an angle of $90^{\circ}$, and they are separated from each other by low-field regions spanning the same angle. The radial component of u has opposite signs on the two halves: pointing inward for $x<0$ and pointing outward for $x>0$. The segmentation of half of the magnet is optimized with our approach, the other half being mirror symmetric. The example shown in Fig. 14(b) corresponds to eight segments per half, for a total of $N=16$ segments. The top panel in Fig. 14(a) shows the value of $\beta$ as a function of $N$, which increases toward the continuous limit as $N \rightarrow \infty$.

It would not be very meaningful to consider the fieldquality figure of merit $\gamma^{2}$ for this case. The vector field $\mathbf{u}(\mathbf{x})$ is not a physically acceptable magnetic field distribution as it violates Ampère's law inside the high-field region, and the parallel component of $\mathbf{u}$ is not conserved across the border between low-field and high-field regions. Despite the lack of these properties, $\mathbf{u}(\mathbf{x})$ can be used to define a valid linear objective functional $\mathcal{S}$ that is a good description of the purpose of this magnetic system. For the examples analyzed above, the figure of merit $\gamma^{2}$ is approximately $75 \%$.

Instead of evaluating the field quality, it is more insightful to present the results in terms of the figure of merit $\Lambda_{\text {cool }}$, which is an evaluator of magnetic systems designed for room-temperature magnetic refrigeration [35]. This parameter quantifies the cooling power of a device using gadolinium as magnetocaloric material. If we denote by $V_{\text {high }}$ the volume of the high-field region and by $V_{\text {mag }}$ the permanent-magnet volume, $\Lambda_{\text {cool }}$ is defined as follows:

$$
\Lambda_{\text {cool }}=\frac{V_{\text {high }}}{V_{\text {mag }}}\left(\left\langle B^{2 / 3}\right\rangle_{\text {high }}-\left\langle B^{2 / 3}\right\rangle_{\text {low }}\right),
$$

where the space averages are evaluated over the highfield and low-field regions of the air gap, respectively. Since this parameter is not normalized by the remanence of the permanent magnet, we use $B_{r}=1.4 \mathrm{~T}$ for this calculation. This value is realistic for modern neodymiumbased permanent magnets. $\Lambda_{\text {cool }}$ as a function of $N$ is shown in the bottom panel in Fig. 14(a). These values are within the range of published designs [35], showing that this approach is successful in insulating the low-field region from the stray field, even though the objective functional does not depend on the field distribution inside the low-field regions. 
As can be seen, the slope of $\Lambda_{\text {cool }}$ with respect to $N$ is quite steep when the number of segments is approximately 10 or less. A realistic cost optimization for such a device must take into account the number of segments besides the total magnet volume.

The configuration shown in Fig. 1 corresponds to the same optimization problem considered in this section. However, the optimal external border of each magnet block is computed with the approach presented in Sec. II E. See the script in Ref. [36]. The real flux-density distribution is shown in the cross sections.

\section{DISCUSSION}

\section{A. Underlying assumptions and optimization objective}

In this section we discuss the peculiarities of the optimization problem considered in the present work, as formulated in Sec. II A. The optimality principles used by our method rely on the linearity of the constitutive B-H relations and the linearity of the objective functional $\mathcal{S}$ with respect to the magnetic field $\mathbf{H}_{1}$.

Assuming a linear objective is equivalent to maximizing point-by-point the field intensity along the desired direction, without penalizing the intensity of the field along the undesired directions. It is clear that such an objective is not suitable for design problems for which the quality of the field distribution is of primary importance. On the other hand such an objective readily describes magnetdesign problems for which the intensity of the field is the main requirement. Moreover, the quality of the field can be increased by performing subsequent optimization procedures aimed at removing the undesired components of the field. One possible approach for this purpose is shimming the bore with high-permeability materials, such as iron $[37,38]$.

As mentioned above, the method is based on assuming a linear constitutive relation for all the materials involved in the simulation. For the iron parts, this approximation is justified as long as the operating point is far below the magnetic saturation regime. In most applications it is undesirable for a magnetic system to operate in the saturation regime, since this leads to distortion of the generated field profile, and the total magnetic reluctance of the system is increased. Moreover, modern permanent magnets are designed to operate around the point where the energy product $\mathbf{H} \cdot \mathbf{B}$ is maximum. In this regime it is is justifiable to approximate the behavior with a linear constitutive relation. Analogously to the iron parts, operating the permanent magnets in the highly nonlinear region of the $\mathbf{B}$ $\mathbf{H}$ relation, corresponding to extremely intense transverse or demagnetization fields, would reduce the performance of the design with respect to both field intensity and field quality [39]. It is also worth stressing that the small deviations caused by the linear approximation have a relatively smaller importance for magnet-design problems aimed at maximizing the intensity of the generated field distribution.

We choose to simulate the permanent magnets using relative permeability $\mu_{r}=1$. This assumption is required only for the purpose of calculating the optimal shape of the interface separating each magnet block from the surrounding air. When the magnet region is predetermined and filled by permanent-magnet material, it is possible to use any value of the permeability. Only for simplicity do we use $\mu_{r}=1$ for all cases, which is not an unreasonable approximation for neodymium-based magnets, for example, and for most modern rare-earth permanent-magnet materials [29].

As mentioned in Sec. II B, we have to assume that the set of all points of $R_{m}$ where $\mathbf{H}_{2}$ shares the same direction is a connected set. Together with the continuity of $\mathbf{H}_{2}$ over $R_{m}$, this condition causes the resulting regions $R_{m}^{(k)}$ to be connected. In some cases it might thus be necessary to apply the procedure to a restricted magnet region, as we did for the examples discussed in Secs. III A and III B.

For the two-dimensional case it would also be possible to use the expedient discussed in Ref. [10]; that is, introducing the accumulated angle. One possible workaround for the three-dimensional case is to use a region-growing algorithm that enforces connectedness of the resulting sets. The $N$ regions are gradually expanded by decreasing the threshold $L_{0}$ defined in Sec. II E until the desired volume is reached. This calculation would have to be repeated at each step of Lloyd's iteration. However, it is important to stress that the connectedness requirement is not naturally embedded into the linear framework adopted here. The analytical arguments for the convergence of Lloyd's method do not apply if connectedness is introduced as an additional requirement rather than spontaneously emerging from a smart choice of $R_{m}$.

As we have seen through different examples, the optimal shapes of the permanent-magnet segments with respect to linear objective functionals are characterized by curved faces. These shapes would be unfeasible for most of the currently used manufacturing processes. However, it would be possible to simplify the optimal shapes into approximated shapes that, while not being perfectly optimal, would be easier to fabricate. Such a possibility will be discussed in future work. It is also worth mentioning that additive manufacturing $[17,40,41]$ might be a very convenient route for manufacturing permanent-magnet blocks with curved boundaries. Knowledge of the optimal solution is not only interesting from a theoretical point of view but is also a useful resource for the purpose of producing an efficient design, especially as the optimal solution for a chosen external boundary can be determined with our method. 
So far we have tacitly assumed the optimal configurations computed with the virtual-magnet method can be realized physically. However, it should be noted that the optimal configurations are not energetically stable with respect to the magnetostatic interaction. The tendency of these dipole-dipole interactions is to minimize the total magnetostatic energy. This tendency corresponds to the objective of minimizing the integral over the whole space of the squared norm of the magnetic field, or equivalently minimizing the integral over $R_{m}$ of the energy density $-\mathbf{M}_{1} \cdot \mathbf{H}_{1}$ [42,43]. If instead we were considering the mutual magnetostatic interaction between the virtual magnet and the real magnet, which corresponds to maximizing the integral of $\mathbf{M}_{2} \cdot \mathbf{H}_{1}$, the configurations computed with our approach would be stable. This aspect, discussed in Ref. [10], can be exploited for automatic alignment of permanent magnets [44] or postassembly magnetization [45]. However, the relevant physical mechanism is the self-interaction of the different parts of the real magnetic assembly, which corresponds to a functional that is quadratic with respect to the real magnetic field $\mathbf{H}_{1}$. The stable equilibrium configurations are characterized by closed magnetization loops that minimize the field external to the magnet. Since the goal of our optimization problem, expressed by a linear functional, is to maximize the average field generated in $R_{g}$ along a desired direction, the optimized configurations are not stable. Mechanically, this instability means that the blocks experience forces and torques trying to rearrange them in stable closed loops [46]. To prevent the rearrangement, the blocks have to be glued or otherwise mechanically constrained in their final configuration.

As mentioned above, the configurations optimized with our approach do not minimize the dipole-dipole interaction energy. Therefore, these configurations are also magnetically unstable. Magnetically, the instability means that the magnetization vectors at points inside the permanentmagnet region $R_{m}$ experience transverse or opposing fields trying to rearrange them in closed loops. This tendency is counteracted by the exchange interaction and the crystal anisotropy [43]. Only when the demagnetizing fields are very strong might some parts of the permanent magnet undergo nonlinear demagnetization, which would reduce the field external to the permanent magnet [39]. However, as discussed above, in many relevant cases the nonlinear effects are not strong enough to affect the behavior of modern permanent-magnet materials, which are strongly anisotropic. In these conditions the magnetic assembly is essentially stable from a magnetic point of view, and the approximation of linear magnetic behavior is thus justified.

\section{B. Structure of the configuration space and optimality}

In this section we discuss the structure of the optimization problem and the optimality of the solutions found by applying Lloyd's method to a randomly generated initial state. In Ref. [27] variational techniques were used to prove that for the two-dimensional case a centroidal Voronoi tessellation is not guaranteed to be unique, not even for $N=2$. Here the two-dimensional case is of relevance since we are partitioning the $\hat{\mathbf{H}}$ sphere; that is, the Voronoi cells are determined from the great-circle distance, which depends only on $\psi$ and $\vartheta$. Our evidence confirms the presence of multiple local solutions.

To illustrate our conclusions, we consider again the optimization problem discussed in Sec. III A. The algorithm is used to subdivide the first octant of the spherical design region using five segments, corresponding to $N=40$ total segments. We perform the optimization procedure on $10^{4}$ different randomly generated initial sets of remanence vectors. The results are shown in Fig. 15. The value of $\mathcal{S}$ normalized by the continuous limit $\mathcal{S}_{\infty}$ and expressed as a percentage is plotted in the top panel as a function of the iteration number.

For this example we find two topologically distinct solutions, labeled as "solution 1" and "solution 2." The corresponding geometries are shown in the bottom panel in Fig. 15. The algorithm converges to the first solution in $91.46 \%$ of cases. For this solution $\mathcal{S} / \mathcal{S}_{\infty}=95.43 \%$. The remaining $8.54 \%$ of the cases converge to the second solution, characterized by $\mathcal{S} / \mathcal{S}_{\infty}=94.60 \%$. As can be seen, in the vicinity of the solution, the rate of variation of $\mathcal{S}$ between consecutive iterations decreases. This feature can also be observed in the vicinity of the value of $\mathcal{S}$ given by solution 2 for cases eventually converging to solution 1. In these cases the trajectory $\mathcal{S}$ (iteration no.) describes a sigmoid shape between the two final values. These results indicate that solution 1 is the globally optimal solution, and that the basin of attraction of this solution is much wider than that of solution 2 .

Some of the traits exhibited by this illustrative problem are found in all the problems we consider. As also observed in Ref. [47], the number of different locally optimal solutions increases with increasing $N$. For small values of $N$ (i.e., those that are practically relevant), we identify a small number of different solutions that are very often topologically distinct from each other. Some of the different solutions for the case in Fig. 11 are shown in Ref. [30].

In most of the cases we find that the solution giving the highest value of $\mathcal{S}$ is also the one most likely to be obtained from a random initial configuration. It is anyway advantageous to repeat the optimization at least a few times to obtain some insight into the particular problem at hand. However, as the framework described above requires only calculation of the virtual field once, these calculations are fast to perform. Only for large values of $N$, which would in all cases be unfeasible, could identifying the global optimum be problematic. Furthermore, the values of $\mathcal{S}$ for the different solutions are very near to each other when 

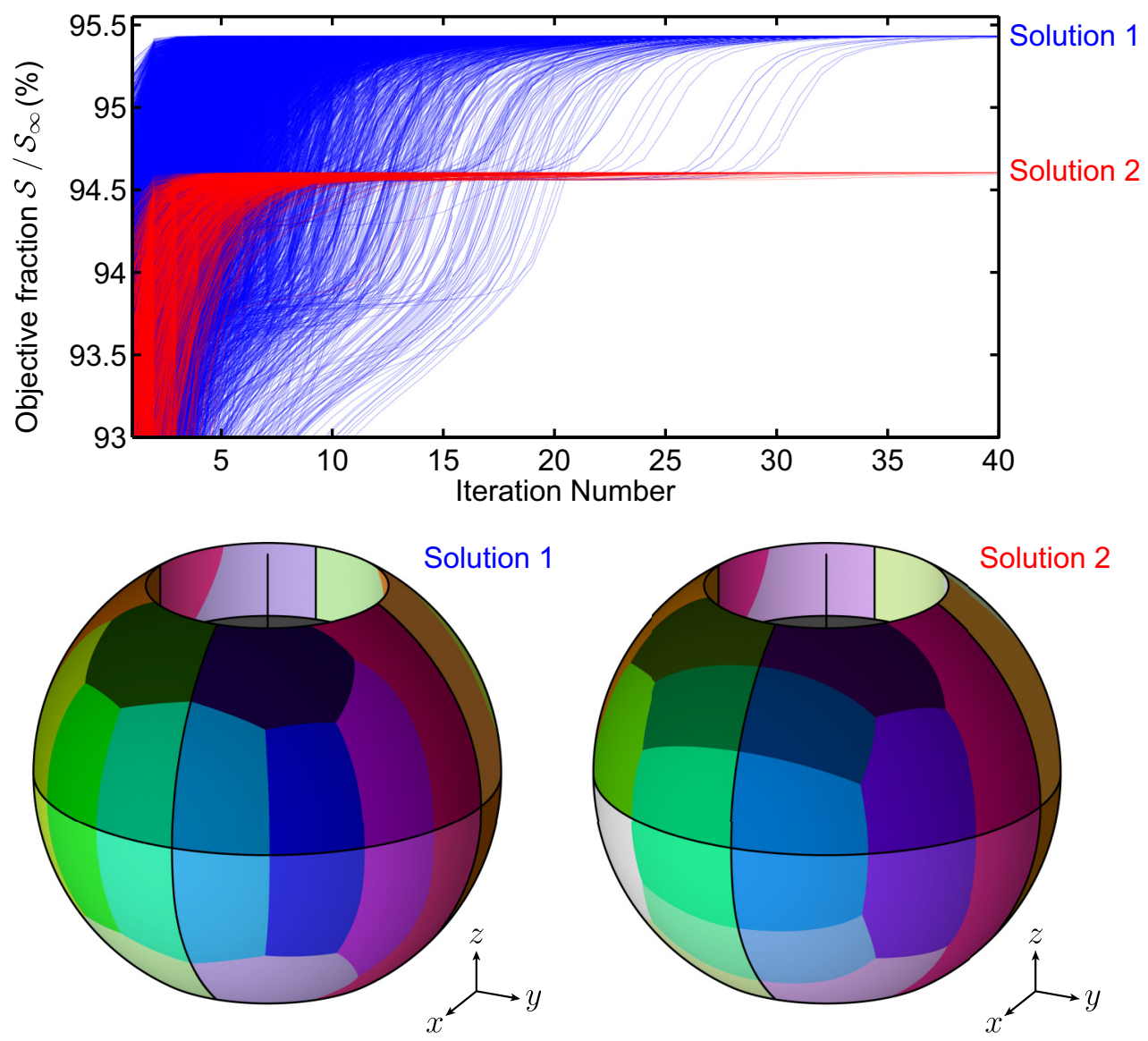

FIG. 15. Results of the algorithm for the optimization problem discussed in Sec. III A, with spherical $R_{m}$. We test Lloyd's method with $10^{4}$ randomly generated initial configurations. The top panel shows the evolution of the objective, expressed as $\mathcal{S} / \mathcal{S}_{\infty}$, as a function of the iteration number. The two topologically distinct solutions are shown in blue and red, respectively. The first solution, obtained in $91.46 \%$ of the cases, gives a slightly larger value of $\mathcal{S}$ and is believed to be the globally optimal solution to this problem. The two corresponding segmented geometries are in the bottom panel.
$N$ is large. If necessary, one could use the more-advanced algorithm proposed in Ref. [47].

\section{Comparison with topology optimization}

As anticipated in Sec. I, the framework of the virtualmagnet method presents both advantages and disadvantages with respect to topology-optimization approaches. Hence, depending on the problem at hand, it might be convenient to choose one method or the other one. The strengths and limits of the two frameworks are listed in Table I.

Since our method is based on the two optimality properties discussed in Sec. II B, the computation time is relatively short even for three-dimensional optimization problems, which would require a much longer computation time when performed with topology-optimization approaches. For the same reason, as discussed in Sec. IV B, the virtual-magnet method is much more likely to provide the globally optimal solution.

With both frameworks, the shape of the resulting uniformly magnetized blocks is not limited to some parameterized geometrical configurations. Moreover, since the field computation can be performed with numerical methods (typically finite-element methods [18,21,22], or the finite-difference method [17]), both approaches can be used with arbitrary geometries, even when the analytical expression of the field distribution is not known. One of the difficulties of topology-optimization approaches is that the resulting shapes might be characterized by irregular or jagged boundaries. Because of these issues, often caused by numerical instabilities, some regularization of the shapes may be required before the blocks are manufactured. Additionally, one of the defining features of topology-optimization approaches is that the resulting magnet pieces might not be simply connected, which in this context is actually a disadvantage. In fact, the presence of non-simply-connected segments severely complicates the manufacturing process unless the resulting shapes are simplified.

In contrast to topology optimization, our approach cannot be used to compute the optimal shape of iron parts: these must be decided in advance. However, it is worth mentioning that a convenient strategy compatible with our framework is to use the level surfaces of the virtual magnetic scalar potential as a border between the permanent magnet and surrounding iron parts. This idea is discussed in Ref. [48].

The two main limitations of the virtual-magnet method when compared with topology optimization are that the 
TABLE I. Comparison between the virtual-magnet method and topology optimization.

\begin{tabular}{lcc}
\hline \hline & Virtual magnet & Topology optimization \\
\hline Short computation time for three-dimensional problems & Yes & No \\
Strong confidence of global optimum & Yes & No \\
Any shape of permanent-magnet blocks & Yes & Yes \\
Applicable for any geometry & Yes & Yes \\
Does not require regularization of shapes & Yes & No \\
Computes optimal shapes of iron parts & No & Yes \\
Applicable for nonlinear objectives & No & Yes \\
Applicable with nonlinear constitutive relations & No & Yes \\
\hline \hline
\end{tabular}

former is not applicable for nonlinear objective functionals and nonlinear $B-H$ constitutive relations. In conclusion, topology optimization is a more-flexible approach, while the virtual-magnet method is generally more efficient and reliable than topology optimization.

\section{CONCLUSION}

We introduce an optimization approach that quickly calculates the optimal segmentation of a three-dimensional given region into uniformly magnetized blocks. Because it relies on the reciprocity theorem, our method significantly reduces the complexity of this optimization problem. By considering a linear magnetic behavior and a linear objective functional, we are able to recast the magnetsegmentation problem into a different well-known mathematical problem: the generation of centroidal Voronoi tessellations. The literature on this topic is abundant: convergence rates and robustness of different algorithms have been investigated [24]. We use Lloyd's method, which is the most straightforward and is completely satisfactory for our purpose.

The framework proposed here offers great insight into the optimization problem, as the optimal configurations are restricted to a countable set of solutions simultaneously satisfying two well-defined optimality properties: the points of each magnet block are the ones giving the best alignment between the virtual field and the remanence vector, which must at the same time be aligned with the average direction of the virtual field over the same region.

These local solutions are often topologically different from each other. Finding the globally optimal solution within this small set is the only part of the problem that must be solved by statistical approaches. When the number of segments is very large, it might be convenient to use advanced techniques, such as the ones presented in Ref. [47]. Generally, when the number of segments is not too large (i.e., for realistic magnet-design problems), a few repetitions already give a fair degree of confidence that the global optimum has been found.

\section{APPENDIX A: CENTROIDAL VORONOI TESSELLATION}

We assume a region $R \subset \mathbb{R}^{n}$ and a set of $N$ points $\left\{\mathbf{x}_{k} \in R\right\}_{k=1, \ldots, N}$ called "generators." We introduce a distance function $d: \mathbb{R}^{n} \times \mathbb{R}^{n} \rightarrow[0,+\infty)$, such that the distance between two points $\mathbf{x}_{a}$ and $\mathbf{x}_{b}$ is given by $d\left(\mathbf{x}_{a}, \mathbf{x}_{b}\right) \geq 0$. The function $d$ must obey the properties of non-negativity, identity of indiscernibles, symmetry, and triangle inequality.

The Voronoi tessellation generated by the set $\left\{\mathbf{x}_{k}\right\}$ is the collection of $N$ regions $\left\{R^{(k)} \subset R\right\}$, called "Voronoi cells," defined by

$$
R^{(k)}=\left\{\mathbf{x} \in R: d\left(\mathbf{x}, \mathbf{x}_{k}\right) \leq d\left(\mathbf{x}, \mathbf{x}_{k^{\prime}}\right) \forall k^{\prime} \neq k\right\} .
$$

We note that $\bigcup_{k} R^{(k)}=R$. We also introduce a density function $\rho(\mathbf{x})$. The centroid of a given set $R^{\prime} \subset R$ is thus given by

$$
\mathbf{x}_{k}^{*}=\frac{\int d V \rho(\mathbf{x}) \mathbf{x}}{\int d V \rho(\mathbf{x})} .
$$

A centroidal Voronoi tessellation is a Voronoi tessellation for which each generator coincides with the centroid of the corresponding cell:

$$
\mathbf{x}_{k}^{*} \equiv \mathbf{x}_{k} \forall k
$$

\section{APPENDIX B: FIGURES OF MERIT}

In this section we introduce a nonlinear functional $\gamma^{2}$ that is used as figure of merit for the field quality. It is convenient to adopt the concise notation typically used in the context of Hilbert spaces:

$$
\begin{aligned}
\mathbf{V} \cdot \mathbf{W} & =\int_{R_{g}} d V \mathbf{V}(\mathbf{x}) \cdot \mathbf{W}(\mathbf{x}), \\
\|\mathbf{V}\|^{2} & =\int_{R_{g}} d V\|\mathbf{V}(\mathbf{x})\|^{2} .
\end{aligned}
$$

We denote by $\mathbf{B}_{1}$ the flux-density distribution of the real system, which inside the air gap is given by $\mathbf{B}_{1}=\mu_{0} \mathbf{H}_{1}$. 
The flux-density distribution $\mathbf{B}_{1}(\mathbf{x})$ can be decomposed into the desired component $\boldsymbol{\Gamma}(\mathbf{x})$ and the field distortion $\Delta(\mathbf{x})$ :

$$
\mathbf{B}_{1}(\mathbf{x})=\boldsymbol{\Gamma}(\mathbf{x})+\boldsymbol{\Delta}(\mathbf{x})=\mathbf{u}(\mathbf{x}) \beta\left[\mathbf{B}_{1}\right]+\boldsymbol{\Delta}(\mathbf{x}),
$$

where the functional $\beta$, which is proportional to $\mathcal{S}$ and thus linear in $\mathbf{B}_{1}$, is given by

$$
\beta\left[\mathbf{B}_{1}\right]=\frac{\mathbf{B}_{1} \cdot \mathbf{u}}{\|\mathbf{u}\|^{2}} .
$$

The value of this functional is used as a figure of merit for the field intensity. If the vector field $\mathbf{u}(\mathbf{x})$ is nondimensional, $\beta$ is expressed in teslas. When $\mathbf{u}(\mathbf{x})=\hat{\mathbf{e}}_{x}$, as for the example shown in Fig. 6, the Hilbert-space squared norm $\|\mathbf{u}\|^{2}$ is equal to the air-gap volume $V_{g}$, and $\beta\left[\mathbf{B}_{1}\right]$ is equal to $\left\langle B_{x}\right\rangle_{R_{g}}$; that is, the $x$ component of the flux density $\mathbf{B}_{1}$ averaged over the air gap $R_{g}$. In this case $\mathbf{u}(\mathbf{x}) \beta\left[\mathbf{B}_{1}\right]$ is a uniform field having the same norm and oriented in the $x$ direction, highlighting the reason why $\boldsymbol{\Delta}(\mathbf{x})$ is the field distortion. By construction, the two vector fields $\boldsymbol{\Gamma}$ and $\boldsymbol{\Delta}$ are orthogonal to each other: $\boldsymbol{\Gamma} \cdot \boldsymbol{\Delta}=0$, where the scalar product is defined by Eq. (B1).

We can now introduce the two nonlinear adimensional functionals $\gamma$ and $\delta$, defined as

$$
\begin{gathered}
\gamma\left[\mathbf{B}_{1}\right]=\frac{\|\boldsymbol{\Gamma}\|}{\left\|\mathbf{B}_{1}\right\|}, \\
\delta\left[\mathbf{B}_{1}\right]=\frac{\|\boldsymbol{\Delta}\|}{\left\|\mathbf{B}_{1}\right\|},
\end{gathered}
$$

which obey the following normalization condition:

$$
\gamma\left[\mathbf{B}_{1}\right]^{2}+\delta\left[\mathbf{B}_{1}\right]^{2}=1 .
$$

The functional $\gamma$ is the Hilbert space analogous to the cosine of the angle $\alpha$ between two vectors, and $\delta$ corresponds to the sine. The value of $\gamma^{2}$ is used to quantify the field quality: when $\gamma^{2}=1$, the field distribution $\mathbf{B}_{1}(\mathbf{x})$ is proportional to the desired distribution $\mathbf{u}(\mathbf{x})$. Physically, $\gamma^{2}$ can be interpreted as the fraction of the air-gap magnetic energy that is stored in the desired components of the field distribution. Equivalently, $\delta^{2}$ is the energy fraction corresponding to the undesired components of the field.

An alternative estimator of the field quality is given by the following expression:

$$
\tau=\frac{\|\boldsymbol{\Delta}\|}{\|\boldsymbol{\Gamma}\|}
$$

As $\gamma$ is analogous to the cosine of the angle $\alpha$ between two elements of the Hilbert space, $\tau$ can be interpreted as the tangent of $\alpha$. When $\mathbf{B}_{1}(\mathbf{x})$ is proportional to $\mathbf{u}(\mathbf{x})$, the tangent $\tau$ is equal to zero; when $\mathbf{B}_{1}(\mathbf{x})$ and $\mathbf{u}(\mathbf{x})$ are orthogonal, $\tau$ goes to infinity. Both $\delta$ and $\tau$ are monotonically increasing functions of $\alpha$ and are thus equivalent ways of quantifying the deviation from the desired field distribution. For the case $\mathbf{u}(\mathbf{x})=\hat{\mathbf{e}}_{x}$, the parameters $\delta$ and $\tau$ correspond, respectively, to

$$
\delta=\left(\frac{\int_{R_{g}}\left\|\mathbf{B}_{1}(\mathbf{x})-\left\langle B_{x}\right\rangle_{R_{g}} \hat{\mathbf{e}}_{x}\right\|^{2} d V}{\int_{R_{g}}\left\|\mathbf{B}_{1}(\mathbf{x})\right\|^{2} d V}\right)^{1 / 2}
$$

and

$$
\tau=\left(\frac{\int_{R_{g}}\left\|\mathbf{B}_{1}(\mathbf{x})-\left\langle B_{x}\right\rangle_{R_{g}} \hat{\mathbf{e}}_{x}\right\|^{2} d V}{\left\langle B_{x}\right\rangle_{R_{g}}^{2}}\right)^{1 / 2} .
$$

[1] J. M. D. Coey, Permanent magnet applications, J. Magn. Magn. Mater. 248, 441 (2002).

[2] K. Yamazaki and Y. Kanou, Shape optimization of rotating machines using time-stepping adaptive finite element method, IEEE Trans. Magn. 46, 3113 (2010).

[3] L. Yan, I. M. Chen, C. K. Lim, G. Yang, W. Lin, and K. M. Lee, Design and analysis of a permanent magnet spherical actuator, IEEE IRS/RSJ Int. Conf. Intell. Robot. Syst. 13, 239 (2008).

[4] B. Biswas, A magnetic quadrupole from rectangular permanent magnets, Nucl. Instrum. Methods Phys. Res. 605, 233 (2009).

[5] B. Diviacco, Performance optimization of pure permanent magnet undulators, Proc. Int. Conf. Particle Accelerators 2, 1590 (1993).

[6] G. Moresi and R. Magin, Miniature permanent magnet for table-top NMR, Concepts Magn. Reson. 19B, 35 (2003).

[7] N. I. Klevets, Synthesis of magnetic systems producing field with maximal scalar characteristics, J. Magn. Magn. Mater. 285, 401 (2005).

[8] N. I. Klevets, Optimal design of magnetic systems, J. Magn. Magn. Mater. 306, 281 (2006).

[9] W. F. Brown, Magnetostatic Principles in Ferromagnetism (North-Holland Publishing Company, Amsterdam, 1962).

[10] A. R. Insinga, R. Bjørk, A. Smith, and C. R. H. Bahl, Globally Optimal Segmentation of Permanent-Magnet Systems, Phys. Rev. Appl. 5, 064014 (2016).

[11] M. G. Abele, Structures of Permanent Magnets (John Wiley and Sons, New York, 1993).

[12] J. H. Jensen and M. G. Abele, Maximally efficient permanent magnet structures, J. Appl. Phys. 79, 1157 (1996).

[13] M. G. Abele, Linear theory of yokeless permanent magnets, J. Magn. Magn. Mater. 83, 276 (1990).

[14] M. G. Abele and H. Rusinek, Optimum design of yokeless permanent magnets, J. Appl. Phys. 67, 4644 (1990).

[15] R. Bjørk, A. Smith, and C. R. H. Bahl, Analysis of the magnetic field, force, and torque for two-dimensional Halbach cylinders, J. Magn. Magn. Mater. 322, 133 (2010).

[16] K. Halbach, Design of permanent multipole magnets with oriented rare earth cobalt material, Nucl. Instrum. Methods 169, 10 (1980). 
[17] C. Abert, C. Huber, F. Bruckner, C. Vogler, G. Wautischer, and D. Süss, A fast finite-difference algorithm for topology optimization of permanent magnets, J. Appl. Phys. 122, 113904 (2017).

[18] W. Kersten, L. Brandl, R. Wagner, C. Huber, F. Bruckner, Y. Hasegawa, G. Wautischer, D. Süss, and D. Sponar, Additive-Manufactured and Topology-Optimized Permanent-Magnet Spin Rotator for Neutron Interferometry, Phys. Rev. Appl. 12, 014023 (2019).

[19] J. S. Choi, J. Yoo, S. Nishiwak, and K. Izui, Optimization of magnetization directions in a 3-D magnetic structure, IEEE Trans. Magn. 46, 1603 (2010).

[20] S. Sato, T. Sato, and H. Igarashi, Topology optimization of synchronous reluctance motor using normalized Gaussian network, IEEE Trans. Magn. 51, 8200904 (2015).

[21] R. Bjørk, C. R. H. Bahl, and A. R. Insinga, Topology optimized permanent magnet systems, J. Magn. Magn. Mater. 437, 78 (2017).

[22] R. Bjørk and A. R. Insinga, A topology optimized switchable permanent magnet system, J. Magn. Magn. Mater. 465, 106 (2018).

[23] J. Lee, Minho Yoon, Tsuyoshi Nomura, and Ercan M. Dede, Topology optimization for design of segmented permanent magnet arrays with ferromagnetic materials, J. Magn. Magn. Mater. 449, 571 (2018).

[24] Q. Du, V. Faber, and M. Gunzburger, Centroidal voronoi tessellations: Applications and algorithms, SIAM Rev. 41, 637 (1999).

[25] Q. Du, M. Gunzburger, and L. Ju, Advances in studies and applications of centroidal voronoi tessellations, Numer. Math. Theory Methods Appl. 3, 119 (2010).

[26] A. R. Insinga, A. Smith, C. R. H. Bahl, K. K. Nielsen, and R. Bjørk, Virtual Magnet Method - Software Library, http://dx.doi.org/10.11583/DTU.9878621 (2019).

[27] J. C. Urschel, On the characterization and uniqueness of centroidal voronoi tessellations, SIAM J. Numer. Anal. 55, 1525 (2017).

[28] A. Sokolov, Clifford algebra and the projective model of Elliptic spaces, arXiv:1310.2713 [math.MG] (2013).

[29] M. Katter, Angular dependence of the demagnetization stability of sintered Nd-Fe-B magnets, IEEE Trans. Magn. 41, 3853 (2005).

[30] See Supplemental Material at http://link.aps.org/supple mental/10.1103/PhysRevApplied.12.064034 for the pseudocode of the optimization algorithm and the figures of locally optimal solutions for the problem in Sec. III A.

[31] This example corresponds to the file SCRIPT_QuadSphere01.m in Ref. [26].

[32] This example corresponds to the file SCRIPT_ExtBorder01.m in Ref. [26].
[33] R. Baartman, Quadrupole shapes, Phys. Rev. ST Accel. Beams 15, 074002 (2012).

[34] D. Eriksen, K. Engelbrecht, C. R. H. Bahl, R. Bjørk, K. K. Nielsen, A. R. Insinga, and N. Pryds, Design and experimental tests of a rotary active magnetic regenerator prototype, Int. J. Refrigeration 58, 14 (2015).

[35] R. Bjørk, C. R. H. Bahl, A. Smith, and N. Pryds, Review and comparison of magnet designs for magnetic refrigeration, Int. J. Refrigeration 33, 437 (2010).

[36] This example corresponds to the file SCRIPT_MagRef01. $m$ in Ref. [26].

[37] M. G. Abele, H. Rusinek, F. Bertora, and A. Trequattrini, Compensation of field distortion with ferromagnetic materials and permanent magnets, J. Appl. Phys. 75, 6990 (1994).

[38] M. G. Abele, W. Tsui, and H. Rusinek, Methodology of pole piece design in permanent magnets, J. Appl. Phys. 99, 08D903 (2006).

[39] A. R. Insinga, C. R. H. Bahl, R. Bjørk, and A. Smith, Performance of Halbach magnet arrays with finite coercivity, J. Magn. Magn. Mater. 407, 369 (2016).

[40] C. Huber, C. Abert, F. Bruckner, M. Groenefeld, O. Muthsam, S. Schuschnigg, K. Sirak, R. Thanhoffer, I. Teliban, C. Vogler, R. Windl, and D. Süss, 3D print of polymer bonded rare-earth magnets, and 3D magnetic field scanning with an end-user 3D printer, Appl. Phys. Lett. 109, 162401 (2016).

[41] C. Huber, C. Abert, F. Bruckner, M. Groenefeld, S. Schuschnigg, I. Teliban, C. Vogler, G. Wautischer, R. Windl, and D. Süss, 3D printing of polymer-bonded rareearth magnets with a variable magnetic compound fraction for a predefined stray field, Nat. Sci. Rep. 7, 9419 (2017).

[42] D. Süss, T. Schrefl, and J. Fidler, Micromagnetics simulation of high energy density permanent magnets, IEEE Trans. Magn. 36, 3282 (2000).

[43] M. d'Aquino, Doctorate thesis, Università degli studi di Napoli "Federico II", 1-155, (2004).

[44] C. K. Chandrana, J. A. Neal, D. Platts, B. Morgan, and P. Nath, Automatic alignment of multiple magnets into Halbach cylinders, J. Magn. Magn. Mater. 381, 396 (2015).

[45] D. G. Dorrell, M.-F. Hsieh, and Y.-C. Hsu, Post assembly magnetization patterns in rare-earth permanent-magnet motors, IEEE Trans. Magn. 43, 2489 (2007).

[46] R. Messina, L. Abou Khalil, and I. Stanković, Selfassembly of magnetic balls: From chains to tubes, Phys. Rev. E 89, 011202 (2014).

[47] L. Lu, F. Sun, H. Pan, and W. Wang, Global optimization of centroidal voronoi tessellation with Monte Carlo approach, IEEE Trans. Vis. Comput. Graph 18, 1880 (2012).

[48] A. R. Insinga, R. Bjørk, A. Smith, and C. R. H. Bahl, Optimally segmented permanent magnet structures, IEEE Trans. Magn. 52, 7210306 (2016). 